\title{
Photo-electric Measurements of Submarine Illumination throughout the Year.
}

\author{
By \\ H. H. Poole, Sc.D., \\ Chief Scientific Officer of the Royal Dublin Society, \\ and \\ W. R. G. Atkins, Sc.D., F.R.S., \\ Head of the Department of General Physiology at the Plymouth Laboratory. \\ With 1 Figure in the Text.
}

Accounts have been given in previous papers $(1,2$, and 3$)$ of measurements of submarine illumination carried out neâr Plymouth by means of photo-electric cells. The present paper records the results so obtained during 1928, together with a description of such modifications of the apparatus as further experience had shown to be desirable.

\section{Use of Opal Glass Diffusing Windows}

In view of the troublesome and uncertain nature of the obliquity and azimuth corrections for photometers with ground-glass windows (3, p. 463), diffusing windows were fitted to all the photometers used on, or after, March 6th, 1928 (Series 24 et seq.). These windows consist of thin glass, opalized on both surfaces, supplied by Messrs. James Hetley \& Co., 35 Soho Square, London. This is the best material that we have found yet for the purpose, since it is permanent, and does not cut off an undue proportion of the light. Its diffusing properties were found to be so good that the only obliquity factor required is that for reflection losses at the front surface of the glass $(2$, p. 184). Thus, the effect of azimuth may be entirely disregarded, and the same-small obliquity correction applied to any photometer when used in air. As will be seen later, the average illumination in the sea seems to be at a rather low angle, about the same as that from a uniform grey sky. The obliquity factor for the water-glass surface at this angle would about compensate for the factor allowing for the reduced reflection loss at a water-glass surface as compared with an air-glass surface. Thus, when the submarine photometer is being standardised by comparison with the deck photometer the readings of both photometers are multiplied by the same 
obliquity factor (2, p. 184) corresponding to the conditions at the time, but when the submarine photometer is submerged the corresponding factor is taken as unity.

The use of this glass reduced the sensitivity of photometer G to direct light to $47 \cdot 5 \%$ of its former value. Photometer L, being, originally, relatively much less sensitive to oblique light, had its direct light sensitivity reduced to $32.5 \%$. As, however, the opal glass had also the effect of reducing the obliquity factors for low-angle light, slightly for G, and considerably for $\mathrm{L}$, the effect of the glass on either photometer when illuminated by ordinary daylight, was to reduce the reading to about one-half. The advantages of the diffusing surface far outweigh this loss of sensitivity.

The opal glasses were placed in front of the existing photometer windows, the apertures of which they covered with considerable overlap. The opals on the submarine photometers $\mathrm{K}$ and $\mathrm{L}$ fitted inside the metal rims surrounding the windows, and were attached with a little "Sirs" wax. The large window of photometer $\mathrm{G}$ was reduced to $5 \mathrm{~cm}$. aperture by a thin metal diaphragm, on the top of which the opal was placed.

With the opal in position and an anode potential of 60 volts, $G$ required 167 metre-candles of perpendicular illumination to pass a current of $10^{-9}$ ampere.

Preliminary tests on the effect of this opal glass on the colour-sensitivity of the photometers shows that it is not important.

\section{New Photometers.}

In September, 1928, the Kunz cell in photometer K was replaced by another potassium hydride argon-filled cell made by the General Electric Co. This is considerably more sensitive than the older type of cell. Moreover, as it was felt that a quite unnecessary factor of safety had been used in designing the windows of the submarine photometers (1, p. 104, or 2 , p. 181), the aperture in this photometer was bored out from about 3 to about $5 \mathrm{~cm}$. in diameter, the metal surrounding it being enamelled white.

This photometer is designated J. Its sensitivity on October 12th was $10^{-9}$ ampere for 0.6 m.c. with anode potential 88 volts. For bright light it is generally used at 3 volts, when its sensitivity, which, like most gas-filled cells is not constant, has been known to vary between 3 and 6 m.c. per $10^{-9}$ ampere. It is proposed to try a method which has been suggested by the makers for improving the constancy of this cell, by passing a sufficient current through it to cause a glow discharge just before use.

About the same date the deck photometer G was replaced by a photo- 
meter, H, containing another vacuum potassium cell which had been in use for some years on shore. This cell had been remounted, and fitted with an opal window instead of the original ground-glass one. It is more than four times as sensitive as $\mathrm{G}$ and only requires 40 m.c. to give $10^{-9}$ ampere with anode potential 60 volts.

\section{Modifications in the Electrical Apparatus.}

It was found that, for work in bad weather, the original apparatus suffered from certain grave defects. In the first place, the collection of so many circuits in a single box, which had been arranged to secure compactness, rendered satisfactory insulation more difficult, so that on very damp days "dark currents" became extremely troublesome. Secondly, this box was very heavy, which made the task of landing it from a small boat on the rocks below the Laboratory at Plymouth a difficult and dangerous one if any sea were running. Embarking it was equally difficult, and the use of any other landing-place involved its carriage over a considerable distance. Thirdly, the sound of the telephone was scarcely loud enough for comfort amid the numerous sounds which occur in a small vessel rolling heavily in a seaway, and, finally, the system of connecting the photometer cables alternately to the hightension battery by means of wander-plugs was unsatisfactory for several reasons.

Accordingly, in October, 1928, the amplifier with its high- and lowtension batteries and also the main high-tension battery were removed from the box. This lightened the latter considerably, and allowed the apparatus remaining in it to be more conveniently arranged. Space was only provided in which one or two aluminium hot-water bottles could be placed if necessary to help in keeping the insulating surfaces dry in damp weather. This is necessary, as leakage currents over the surface of the ebonite potentiometer panel will cause "dark currents" even if the effects of extraneous leakage currents are eliminated by means of guard sheets. The design of the potentiometer is such that it would be very difficult to eliminate these internal leakage currents. For this reason it is better to disconnect the standard cell (which is not shown in the figure) from the potentiometer when taking readings under bad conditions.

The figure shows the general lay-out of the apparatus, as modified. $\mathrm{A}$ is the two-valve amplifier, which is of a more powerful type than that previously used. The input terminals are protected from the possibility of surface leakage from the batteries by guard rings of tin-foil gummed to the ebonite panel. It is most important that the telephones, $\mathrm{T}$, should be connected on the negative side of the high-tension battery, 


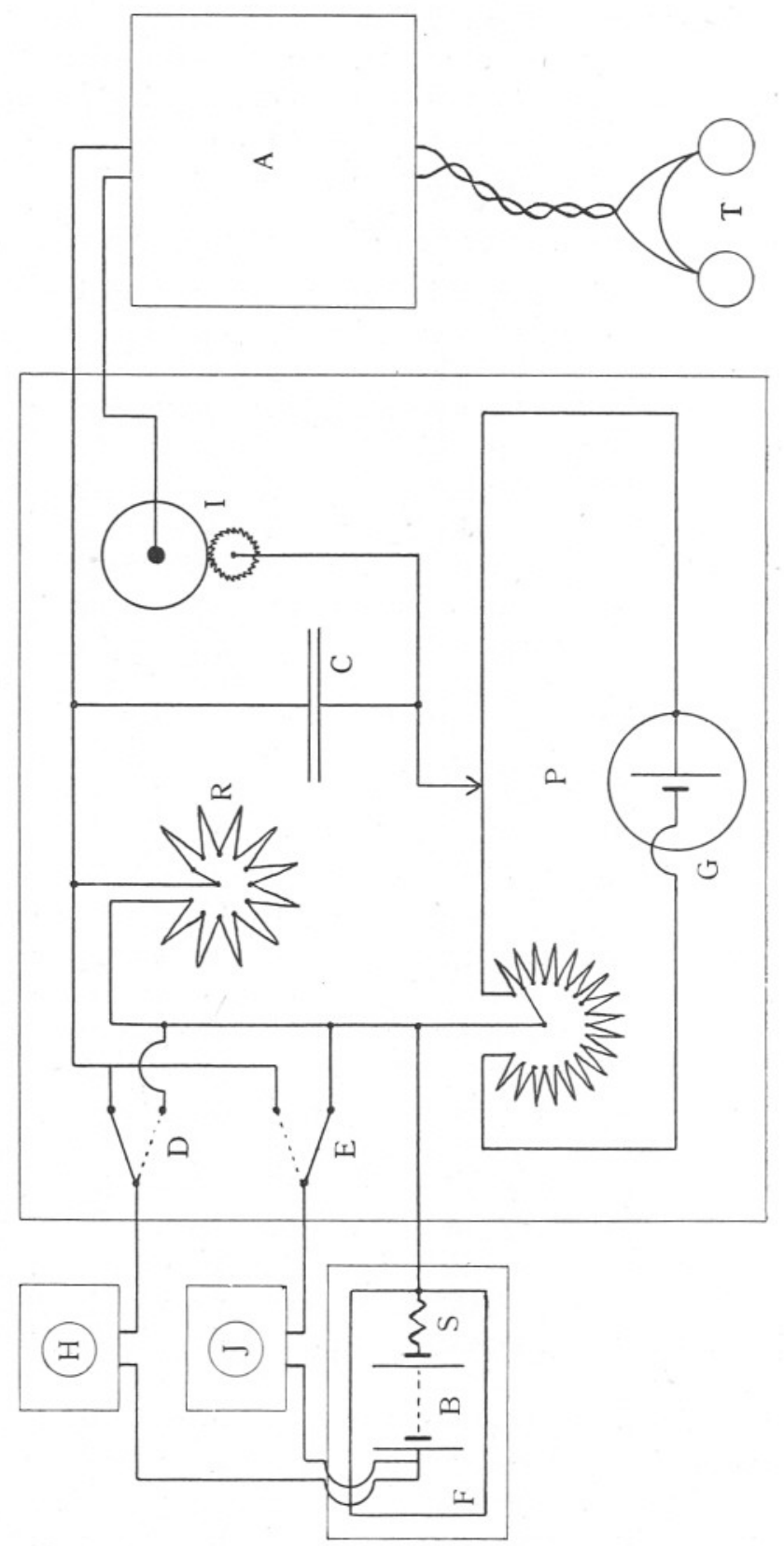


as otherwise a comparatively large P.D. may occur between the observer and the potentiometer, causing very serious " dark currents."

$\mathrm{B}$ is the anode battery, consisting of two 60 -volt H.T. dry batteries. This is mounted on rubber insulators inside a metal box, F, which, in turn, is similarly insulated from the wooden box which surrounds and protects the whole. $\mathrm{F}$ is connected to the negative pole of the battery through a safety resistance, $\mathrm{S}$, which consists of a bobbin resistance of 9,995 ohms made for a Crompton "All Test" voltmeter. F also carries two insulated terminals, to which the anode cables of the photometers, $\mathrm{H}$ and $\mathrm{J}$, are connected. Either of these terminals can be connected by means of a wander-plug to any suitable tapping on B. This arrangement is mechanically much more satisfactory than the direct attachment of the heavy cables to the battery by means of wander-plugs. The effects of leakage currents along the surfaces of the cables are eliminated, as before, by guard wires twisted tightly round the cables and connected to F. These are not shown in the figure.

$\mathrm{D}$ and $\mathrm{E}$ are two 2-way switches to which the negative leads from $\mathrm{H}$ and $\mathrm{J}$ are connected. With these in the positions shown in the figure the current through $\mathrm{H}$ passes through the $100,000 \mathrm{ohm}$ resistance $\mathrm{R}$, and is measured by the potentiometer $\mathrm{P}$, while that through $\mathrm{J}$ flows directly back to $\mathrm{F}$ and the negative terminal of $\mathrm{B}$. To measure the current through $\mathrm{J}$ it is only necessary to reverse the switches. This arrangement enables successive readings to be taken very quickly, and ensures that the potential is applied to the photometers and cables the whole time, so that soakage effects are eliminated. When readings of a gas-filled photometer have to be taken at two different anode potentials at a given depth, it is better to compare the reading with one potential with the reading of the deck photometer, and then repeat the comparison with the new potential, sufficient time - say, several minutes-having elapsed to prevent errors due to soakage. Well-insulated electric light "corrider" switches are very suitable for use at D and E. They are mounted on an ebonite panel which also carries the terminals to which the cables are connected.

$\mathrm{R}$ is generally used at $100,000 \mathrm{ohms}$, but can be set to any lower multiple of $10,000 \mathrm{ohms}$ which may be convenient for measuring larger currents. The capacity of the mica-insulated condenser $\mathrm{C}$ is $1 \mu \mathrm{F}$. The interrupter I has already been described (3, p. 458).

As the terminals of $\mathrm{R}$ are connected to points near the positive end of the potentiometer, the possibility of external leakage from the negative end of the potentiometer battery was eliminated by mounting it on an insulated guard plate $\mathrm{G}$ connected to its positive terminal. The standard cell and the amplifier also stand on guard plates (not shown in the figure) which are connected to G. 


\section{Results.}

The results obtained are shown in Table I, which is a continuation of Table II of our 1928 paper, the various symbols having the same significance. The work was mostly carried out at International Hydrographic Station E1, which is about 10 miles S.W. of the Eddystone lighthouse and about 20 miles from the Plymouth Sound breakwater. As before, the values of the vertical absorption coefficient, $\lambda$, for the layer between 0 and $10 \mathrm{~m}$., which are printed opposite the mean depth, $5 \mathrm{~m}$., are marked with an asterisk to show that a surface loss of light of $15 \%$ has been assumed. The other values of $\lambda$ are obtained from the values of the percentage illumination, $\mathrm{p}$, for the levels immediately above and below, respectively. In a few cases the mean of these depths differs somewhat from that opposite to which $\lambda$ is printed. The vertical illumination is tabulated in thousands of metre candles (k.m.c.).

Series 25 resembles Series 15 in that the photometer window was vertical, so as to measure horizontal light. They differ in the fact that in Series 25 opal glass was used. The effect of this is seen when we compare $p_{h}$ of Series 25 with the corresponding value of $\mathrm{p}$ in Series 24 . The ratio $\mathrm{p}_{\mathrm{h}} / \mathrm{p}$ is given in the last column of Series 25 , where it will be seen that its mean value is as high as 0.54 , as compared with 0.14 in Series 15-16. The latter value is known to be too low, owing to the small sensitivity of the photometer to oblique light, but the intensity of the horizontal illumination in Series 25 is rather surprising. Too much stress must not be laid on this result, as more than two hours elapsed between the two series, and the water may have been clearer in the later one, although the approximate uniformity of the ratio $\mathrm{p}_{\mathrm{h}} / \mathrm{p}$ at different depths may be taken as evidence against this.

It is obvious that in air, under a perfectly uniform, sunless sky, the value of $\mathrm{H} / \mathrm{V}$ would be 0.5 , the vertical photometer only receiving light from half a hemisphere. Thus, the results of Series 24 and 25 indicate that the average obliquity of submarine illumination is about the same as that of diffuse daylight in air. The previous results corresponded more closely with the same light after refraction in a smooth-water surface, which would considerably increase its average altitude (2, p. 184, table). The average altitude of the submarine light has very little effect on the sensitivity to vertical light of a photometer with a diffusing window, as the effect of obliquity on the small reflection loss at the water-glass surface is negligible. Thus, the values given for $\mathrm{V}$ are not affected. On the other hand, the ratio of the total illumination, I, to $\mathrm{V}$ would be 2.0 for light of the distribution suggested by Series 24 and 25, as compared with $1 \cdot 19$ for that assumed from the results of Series 15 and 16 (2, loc. cit.). As the rate of photosynthesis depends on the total illumination, the average altitude of the light is of importance, and further measurements are desirable. 


\section{TABLE I.}

Date, Remarks, etc.

Serifs 23. 1.3.'28.

K Photometer. At E1.

Wind S.W., light. Long swell, small surface rip. ples. Clear sky. Approx. depth $70 \mathrm{~m}$. (Chart). High water 12.59 p.m. Secchi disc $14 \mathrm{~m}$. in shadow of ship at 4.30 p.m.

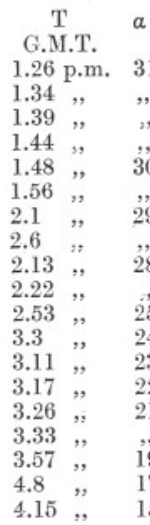

SERIES 24. 6.3.'28.

L Photometer. Ahout 1.5 miles N.W. of Eddy. stone, drifting slowly S.W. Depth 51 m. 12.57 (Sounding). Wind N.W., 1.1, light. Very little swell, surface ripples. Sky clear. High water 6.20 p.m. Secchi dise $13.5 \mathrm{~m}$. in shadow of ship at 2.40 p.m., $11.5 \mathrm{~m}$. at 2.50 in sun.

Series 25. 6.3.'28.

I Photometer with window vertical so as to measure horizontal light. Sun through light clouds. Other conditions as in Series 24 .

$\mathrm{K}$ Photometer. At El. 12.34 p.m. $\quad 34$

12.39

34

Light.
Bright Sun
",
",
,
,
,
,
,
,
,
,
,
,
",

\begin{tabular}{|c|c|c|}
\hline$\beta$ & d & $V_{\mathrm{a}}$ \\
\hline & metres & k.m.c. \\
\hline 4 & $\mathbf{r}$ & \\
\hline ,", & 5 & 66.5 \\
\hline , & 10 & 66.5 \\
\hline & 15 & 66.5 \\
\hline "’ & 20 & 65 \\
\hline & 25 & $64 \cdot 5$ \\
\hline 3.5 & 30 & 65 \\
\hline ", & 35 & $65 \cdot 5$ \\
\hline & 40 & 64 \\
\hline$"$ & 45 & $62 \cdot 5$ \\
\hline & 50 & $61 \cdot 5$ \\
\hline & 55 & 54 \\
\hline 3 & 60 & $52 \cdot 5$ \\
\hline & 70 & $\begin{array}{l}50 \\
48.5\end{array}$ \\
\hline & 45 & 47.5 \\
\hline & 20 & $40 \cdot 5$ \\
\hline $2 \cdot 4$ & 5 & $38 \cdot 3$ \\
\hline & $\mathrm{r}$ & $33 \cdot 8$ \\
\hline
\end{tabular}

V
k.m.c.
66.5
$26 \cdot 1$
$15 \cdot 7$
$9 \cdot 6$
$5 \cdot 75$
$3 \cdot 34$
$2 \cdot 09$
$1 \cdot 30$
$0 \cdot 75$
$0 \cdot 42$
$0 \cdot 200$
$0 \cdot 090$
$0 \cdot 036$
$0 \cdot 011$
$0 \cdot 008$
$0 \cdot 175$
$2 \cdot 52$
$10 \cdot 1$
$33 \cdot 8$

$\begin{array}{cc}\mathrm{p} & \lambda \\ \mathrm{o} & \\ 100 & - \\ 39 \cdot 4 & 0 \cdot 127 \\ 23 \cdot 7 & 0 \cdot 103 \\ 14 \cdot 4 & 0 \cdot 099 \\ 8 \cdot 85 & 0 \cdot 103 \\ 5 \cdot 15 & 0 \cdot 101 \\ 3 \cdot 22 & 0 \cdot 094 \\ 2 \cdot 00 & 0 \cdot 101 \\ 1 \cdot 17 & 0 \cdot 109 \\ 0 \cdot 67 & 0 \cdot 127 \\ 0 \cdot 326 & 0 \cdot 138 \\ 0 \cdot 167 & 0 \cdot 156 \\ 0 \cdot 068 & 0 \cdot 198 \\ 0 \cdot 023 & 0 \cdot 144 \\ 0 \cdot 016 & - \\ 0 \cdot 357 & - \\ 6 \cdot 2 & - \\ 26 \cdot 4 & - \\ 100 & -\end{array}$

Bright Sun

$\begin{array}{rr}3 & 5 \\ , & 10 \\ " & 15 \\ , & 20 \\ , & 25 \\ , & 30\end{array}$

75

$73 \cdot 5$

73

$73 \cdot 5$

$72 \cdot 5$

$71 \cdot 5$

$30 \cdot 9$

$13 \cdot 7$

$6 \cdot 35$

$3 \cdot 03$

1.47

$0 \cdot 63$

$0 \cdot 31$

$\begin{array}{ll}41 & 0 \cdot 152 * \\ 18 \cdot 6 & 0 \cdot 155 \\ 8 \cdot 7 & 0 \cdot 151 \\ 4 \cdot 1 & 0 \cdot 147 \\ 2 \cdot 0 & 0 \cdot 155 \\ 0.87 & 0 \cdot 152 \\ 0.435 & -\end{array}$

3.12 p.m. 24 $3.17, \quad 23$ 3.20 , $3.31, \quad 22$ 3.35 ,

2

Weak Sun

Brighter

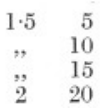

2
,$\quad 25$
$18 \cdot 5$

$18 \cdot 35$

$19 \cdot 7$

$29 \cdot 6$

$29 \cdot 6$
H

$4 \cdot 65$

1.90

$0 \cdot 90$

0.57

$0 \cdot 33$ $\underline{p_{h}}$

$\mathrm{ph}$
0.1

$10 \cdot 3 \quad 0.555$

$4 \cdot 55 \quad 0 \cdot 525$

$1.92 \quad 0.47$

$1 \cdot 11 \quad 0.555$
Wind N.W. light. Con. siderable swell. Over. cast, grey sky. High water $9.59 \mathrm{p} . \mathrm{m}$.

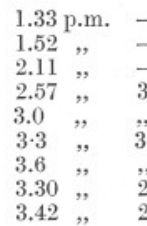

Series 27. 5.4.'28. 1.2 p.m. 44

K Photometer. At El. 1.13

Light breeze S.W. Very heavy roll at times. Blue sky to E. Heavy clouds to W. Light variable. High water 6.9 p.m. Sechi dise $10 \mathrm{~m}$. in shadow of ship at 4.15 p.m.

$\begin{array}{ccc}\mathrm{V} & \mathrm{p} & \lambda \\ 39 \cdot 3 & 100 & - \\ 18 \cdot 0 & 34 \cdot 3 & 0 \cdot 172 \\ 6 \cdot 65 & 15 \cdot 1 & 0 \cdot 126 \\ 6 \cdot 25 & 9 \cdot 5 & 0 \cdot 117 \\ 3 \cdot 02 & 4 \cdot 7 & 0 \cdot 145 \\ 1 \cdot 42 & 2 \cdot 22 & 0 \cdot 162 \\ 0 \cdot 615 & 0 \cdot 925 & 0 \cdot 160 \\ 0 \cdot 288 & 0 \cdot 45 & 0 \cdot 127 \\ 0 \cdot 060 & 0 \cdot 137 & - \\ & & \\ 67 & 100 & - \\ 21 \cdot 4 & 41 & 0 \cdot 139 \\ 10 \cdot 2 & 21 \cdot 2 & 0 \cdot 128 \\ 4 \cdot 65 & 11 \cdot 3 & 0 \cdot 129 \\ 1 \cdot 68 & 5 \cdot 8 & 0 \cdot 144 \\ 0 \cdot 76 & 2 \cdot 66 & 0 \cdot 132 \\ 0 \cdot 505 & 1 \cdot 54 & 0 \cdot 092 \\ 0 \cdot 345 & 1 \cdot 06 & 0 \cdot 103 \\ 0 \cdot 206 & 0 \cdot 55 & 0 \cdot 115 \\ 0 \cdot 134 & 0 \cdot 335 & 0 \cdot 108 \\ 0 \cdot 104 & 0 \cdot 192 & 0 \cdot 135 \\ 0 \cdot 149 & 0 \cdot 173 & -\cdot \cdot \\ 0 \cdot 065 & 0 \cdot 087 & 0 \cdot 129 \\ 0 \cdot 036 & 0 \cdot 051 & - \\ 2 \cdot 83 & 4 \cdot 25 & - \\ 8 \cdot 9 & 14 \cdot 8 & - \\ 58 & 100 & -\end{array}$


$\stackrel{\text { T }}{\text { G.M.T. }} \quad a \quad$ Light.

SERIES 28. 19.4.'28.

11.58 a.m. 51 Bright Sun

K Photometer. At E1. 12.8 p.m. ,"

Wind N.W., light. Slight 12.21 ", ",

swell from N.W. Clear 12.31 ,,

sky with clouds, High 12.40 ,"

water 5.23 p.m. Secchi 12.50 dise $18 \mathrm{~m}$. at 4.18 p.m.

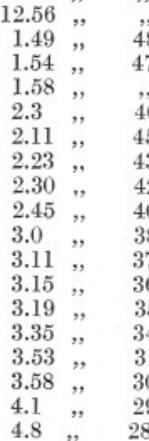

SERIES 29, 7.5.'28.

11.13 a.m. 55 Sun

K Photometer. At E1. 11.29

Very light air from N. 11.32,"

Scarcely any swell, sea 11.46 ,

glassy. Sky hazy with 11.52 ,, , ,

light elouds and hot sun. 11.56

High water 7.13 p.m. 12.2 p.m.

Secchi dise $17 \mathrm{~m}$. in 12.14,

shadow on weather side 12.25

of ship at 2.58 p.m., $19 \mathrm{~m}, 12.29$

in sun on lee side of ship 12.35 ,

at 3.4 p.m. $\left(V_{3}=71,000 \quad 12.56\right.$,

$\mathrm{m} . \mathrm{m}, \mathrm{m}$, to windward $12.59^{\circ}$,

at $4.0 \mathrm{p} . \mathrm{m}$. and $22 \mathrm{~m}$. to 1.13

leeward at 4.5 p.m. with 1.21 ,"

overcast sky $\left(V_{a}=18,700 \quad 1.28\right.$, m.c.).

$1.28, \quad 54$

$1.41, ", \quad 33, "$

$1.46,, \quad, \quad$,

$1.55, \quad 52 \quad$,

$2.34, \quad 47$

2.49,

Sun

Bright Sun

,",

$$
\text { ," }
$$$$
\text { , }
$$

",

,

,

,

,"

",

Cloudy

\section{Light"Cloud}

12.26 p.m.

SERIES $30,13,6,28$

L Photometer. At E1. 12.33 ,"

Wiud S.E., light. Sea 12.40 ,"

smooth with slight swell, 12.45 ",

Sky overcast. High 12.49

water 1.23 p.m.

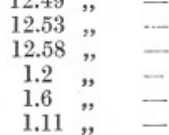

Shries 31, 13.6,'28.

K Photometer. Other conditions as in Series 30.

Sechi dise $14 \mathrm{~m}$, at 3.17 p.m. in lee of ship, $11 \mathrm{~m}$. at 3.22 p.m. to windward of ship.
1.32 p.m. - Variable

1.40 ,

1.47 ,

2.9

$2 \cdot 20$

2.29

). 36 ,

2.52 "

$3 \cdot 3$,

$3.11 "$ -

no Sun
Bright, no Sun 1

\section{,}

,

,$$
\text { , }
$$

,

",
",
",
,

\begin{tabular}{|c|c|c|c|}
\hline$\beta$ & $\begin{array}{c}\mathrm{d} \\
\text { metres }\end{array}$ & $\begin{array}{c}V_{\mathrm{a}} \\
\text { k.m.c. }\end{array}$ & $\begin{array}{c}\mathrm{V} \\
\text { k.m.c. }\end{array}$ \\
\hline 4 & $\mathrm{r}$ & 109 & 109 \\
\hline & a & 114 & 109 \\
\hline 2 & 5 & $45 \cdot 5$ & $22 \cdot 7$ \\
\hline & 10 & 49 & $17 \cdot 2$ \\
\hline 3.5 & $"$ & 81 & $26 \cdot 7$ \\
\hline 4 & $\ddot{\prime}$ & 121 & 41 \\
\hline " & 15 & 134 & $29 \cdot 6$ \\
\hline , & & 102 & $24 \cdot 6$ \\
\hline "' & 20 & 100 & $15 \cdot 6$ \\
\hline " & 25 & 100 & $10 \cdot 4$ \\
\hline$"$ & 30 & 99 & $7 \cdot 35$ \\
\hline 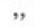 & 35 & 97 & $4 \cdot 15$ \\
\hline$"$ & 40 & 94 & $2 \cdot 12$ \\
\hline & 45 & 94 & $1 \cdot 20$ \\
\hline , & 50 & 89 & 0.65 \\
\hline ", & 55 & 84 & 0.342 \\
\hline 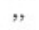 & 60 & $79 \cdot 5$ & $0 \cdot 192$ \\
\hline , & 65 & 78.5 & 0.116 \\
\hline & 70 & $77 \cdot 5$ & 0.076 \\
\hline $3 \cdot 5$ & 45 & 74 & 0.73 \\
\hline " & 25 & $73 \cdot 5$ & $5 \cdot 8$ \\
\hline 1 & " & $25 \cdot 3$ & $1 \cdot 77$ \\
\hline 3 & 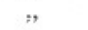 & $69 \cdot 5$ & $5 \cdot 55$ \\
\hline$"$ & $\mathbf{r}$ & 68 & 68 \\
\hline
\end{tabular}

$p$
$\%$
100
$95 \cdot 5$
50
$35 \cdot 1$
$33 \cdot 0$
$33 \cdot 8$
$22 \cdot 1$
$24 \cdot 0$
$15 \cdot 6$
$10 \cdot 4$
$7 \cdot 4$
$4 \cdot 25$
$2 \cdot 25$
$1 \cdot 28$
$0 \cdot 725$
$0 \cdot 405$
$0 \cdot 242$
$0 \cdot 148$
$0 \cdot 098$
$0 \cdot 985$
$7 \cdot 9$
$7 \cdot 0$
$8 \cdot 0$
100

$\lambda$

-

$0 \cdot 090^{*}$

$0 \cdot 077$

0.077

0.077

$0 \cdot 078$

$0 \cdot 078$

$0 \cdot 079$

$0 \cdot 075$

$0 \cdot 088$

$0 \cdot 119$

$0 \cdot 121$

$0 \cdot 114$

$0 \cdot 115$

$0 \cdot 109$

$0 \cdot 100$

0.090

-

-$$
100
$$$$
90 \cdot 5
$$

$74 \cdot 5$

$47 \cdot 5$

$32 \cdot 3$

$23 \cdot 2$

$14 \cdot 2$

8.65

$5 \cdot 15$

$3 \cdot 22$

$1 \cdot 82$

0.955

$0 \cdot 67$

$0 \cdot 385$

$0.220 \quad 0.085$

$0 \cdot 164 \quad 0 \cdot 060$

$0 \cdot 121$

$0 \cdot 340$

$0 \cdot 54$

1.73

$13 \cdot 8$

100

$10 \cdot 45$

53.5

100

$\begin{array}{lrr}69 \cdot 5 & 69 \cdot 5 & 100 \\ 61 & 48 \cdot 5 & 79 \cdot\end{array}$

$56.5 \quad 20 \cdot 4 \quad 36.1$

$\begin{array}{lll}56 \cdot 5 & 20 \cdot 4 & 36 \cdot 1 \\ 52 & 11 \cdot 7 & 22 \cdot 5\end{array}$

$\begin{array}{lr}52 \cdot 5 & 6.55\end{array}$

$53 \quad 3 \cdot 73$

$46 \quad 1.84$

$41 \cdot 5 \quad 0 \cdot 71$

$47 \quad 0 \cdot 46$

$22 \cdot 5$

$7 \cdot 05$

$4 \cdot 00$

$1 \cdot 71$

0.98

0.535

$0.097 *$

0.070

$0 \cdot 083$

$0 \cdot 099$
$0 \cdot 101$

$0 \cdot 099$

$0 \cdot 104$

0.122

$0 \cdot 100$

0.091
0.111

-

=

$0 \cdot 26$

100

$\begin{array}{lccc}34 \cdot 4 & 34 \cdot 4 & 100 & - \\ 46 & 19 \cdot 9 & 43 \cdot 5 & 0 \cdot 105^{*} \\ 40 & 11 \cdot 9 & 29 \cdot 7 & - \\ 48 \cdot 5 & 1 \cdot 77 & 3 \cdot 65 & 0 \cdot 106 \\ 40 & 0 \cdot 495 & 1 \cdot 24 & 0 \cdot 122 \\ 33 \cdot 3 & 0 \cdot 106 & 0 \cdot 32 & 0 \cdot 115 \\ 30 \cdot 2 & 0 \cdot 038 & 0 \cdot 125 & - \\ 28 \cdot 7 & 1 \cdot 87 & 6 \cdot 5 & - \\ 25 \cdot 6 & 10 \cdot 3 & 40 \cdot 0 & - \\ 48.5 & 48.5 & 100 & -\end{array}$

0.133 *

$0 \cdot 106$

0.116

0.114

$0 \cdot 141$

$0 \cdot 141$
0.166

$\begin{array}{lr}\text { l } & \text { r } \\ , & 5 \\ , & 10 \\ , & 30 \\ , & 40 \\ , & 50 \\ , & 60 \\ , & 25 \\ , & 10 \\ , & \text { r }\end{array}$

$48 \cdot 5$ 
Date, Remarks, etc.

SERIES 32. 4.7.'28.

L Photometer. At E1.

Wind S.W., freshening.

Moderate swell, surface

waves breaking. Bright

sun with a few low clouds.

High water 6.36 p.m.

Secchi disc $11 \mathrm{~m}$. at 12.8

p.m. in shadow of ship,

$11.5 \mathrm{~m}$. at $12.14 \mathrm{p} . \mathrm{m}$. in

$\operatorname{sun}\left(V_{\mathrm{a}}=128,000\right.$ m.c.).

Series 33. 11.7'

L Photometer. At E1.

Very little wind. Light

variable, owing to passage

of light clouds across sun.

High water 11.40 a.m.

11.55 a.m. 62

12.13 p.m.

12.20 ,

12.28,

2.39

12.47,

12.59 ,"

Series 34. 11.7.'28.

K Photometer. Other Water surface glassy at end of series. Secchi disc $18.5 \mathrm{~m}$. at $3.28 \mathrm{p} . \mathrm{m}$. in shadow of ship, $16 \mathrm{~m}$. at 3.33 p.m. in sun $\left(V_{a}=\right.$ 76,000 m.c.). conditions as in Series 33.

\begin{tabular}{|c|c|c|c|}
\hline \multirow{2}{*}{\multicolumn{2}{|c|}{1.19 p.m. }} & 59 & \multirow{3}{*}{$\begin{array}{l}\text { Bright Sun. } \\
\text { Light clouds } \\
\text { at times }\end{array}$} \\
\hline & & 58 & \\
\hline 1.46 &, & 57 & \\
\hline 1.52 & ", & 56 & , \\
\hline 1.59 & ", & & ," \\
\hline 2.6 & ," & 55 & , \\
\hline 2.18 & , & 54 & , \\
\hline 2.30 & , & 52 & " \\
\hline 2.38 & ," & 51 & , \\
\hline 2.47 & $"$ & 50 & ", \\
\hline 2.58 & ", & 49 & \\
\hline 3.12 & ," & 47 & ", \\
\hline 3.23 & ," & 45 & " \\
\hline
\end{tabular}

11.36 a.m. $\quad 58$

Series 35. 23.7.'28.

L Photometer. At E1. 11.52,

Wind W., light. Sea 12.4 p.m. ,

calm. Sky clear with 12.8 ,

light clouds low on the 12.16 ,"

horizon. High water 12.20 ,"

9.52 a.m.

$12.29 "$

12.34,

12.37,

Serrivs 36. 23.7''28.

12.52 p.m. $\quad 59$

K Photometer. Other 12.57 , 58

conditions as in Series 35 .

Secchi disc $17.5 \mathrm{~m}$. in

shadow and lee of ship at

3.26 p.m., $14 \mathrm{~m}$. in sun to

windward.

Bright Sun
,,
,,
,
,
,
,

$\begin{array}{lll}1.9, & & , \\ 1.24, & 5 \\ 1.30, & \\ 1.40, & 5 \\ 2.0, & 5 \\ 2.17, & 5 \\ 2.24, & 5 \\ 2.28, & 5 \\ 2.34, & 4 \\ 2.46 & , & 4 \\ 2.58, & 4 \\ 3.19, & 4\end{array}$

$\begin{array}{cclc}\beta & \begin{array}{c}\mathrm{d} \\ \text { metres }\end{array} & \begin{array}{c}\mathrm{V}_{\mathrm{a}} \\ \text { k.m.c. }\end{array} & \begin{array}{c}\mathrm{V} \\ \text { k.m.c. }\end{array} \\ 6 & \mathrm{r} & 126 & 126 \\ \Rightarrow & 5 & 123 & 43 \cdot 8 \\ \Rightarrow & 10 & 122 \cdot 5 & 21 \cdot 8 \\ , & 15 & 122 & 9 \cdot 9 \\ , & 20 & 122 \cdot 5 & 5 \cdot 2\end{array}$

\begin{tabular}{cc}
$p$ & \multicolumn{1}{c}{$\lambda$} \\
$\%$ & \\
100 & - \\
$35 \cdot 6$ & $0 \cdot 156^{*}$ \\
$17 \cdot 8$ & $0 \cdot 148$ \\
$8 \cdot 1$ & $0 \cdot 143$ \\
$4 \cdot 25$ & -
\end{tabular}

Bright Sun.

Light clouds

at times

,",

,

,

,

Bright Sun

,",

,",

,

,

,"

,

",

,'

,

,

,"

$\begin{array}{ccc}3 & \mathrm{r} & 130 \cdot 5 \\ , & 5 & 126 \cdot 5 \\ 2 & 10 & 95 \cdot 5 \\ 2 \cdot 5 & 15 & 113 \\ 3 & 20 & 139 \cdot 5 \\ 2 \cdot 5 & 25 & 105 \cdot 5 \\ , & 30 & 104 \cdot 5 \\ , & 35 & 108 \cdot 5\end{array}$

$130 \cdot 5$
53
$23 \cdot 7$
$16 \cdot 0$
$8 \cdot 7$
$2 \cdot 90$
$2 \cdot 24$
$1 \cdot 32$

$\begin{array}{cc}2 \cdot 5 & \mathrm{r} \\ , & 5 \\ , & 10 \\ 3 & 20 \\ , & 25 \\ 2 \cdot 5 & 30 \\ , & 35 \\ , & 45 \\ , & 55 \\ , & 65 \\ , & 35 \\ 2 & 25 \\ ,, & \text { r }\end{array}$

99
$109 \cdot 5$
114
$115 \cdot 5$
115
111
$107 \cdot 5$
$109 \cdot 5$
108
95
$94 \cdot 5$
82
87

99

$52 \cdot 5$

$43 \cdot 2$

$14 \cdot 5$

$6 \cdot 85$

$3 \cdot 66$

1.76

$0 \cdot 55$

$0 \cdot 194$

0.046

$1 \cdot 09$

$4 \cdot 1$

87

$$
\begin{array}{cr}
4 \cdot 5 & \text { r } \\
, & \mathrm{a} \\
, & 5 \\
, & 10 \\
, & 15 \\
, & 20 \\
, & 25 \\
, & 30 \\
, & 35
\end{array}
$$

118

121

121

119

120

$120 \cdot 5$

$120 \cdot 5$

$121 \cdot 5$

118

$118 \cdot 5$

$60 \cdot 5$

$40 \cdot 2$

$26 \cdot 6$

$12 \cdot 6$

4.45

$1 \cdot 26$

$119 \cdot 5 \quad 119 \cdot 5$

$\begin{array}{ll}119 \cdot 5 & 119 \cdot 5 \\ 120 & 121\end{array}$

$\begin{array}{lr}119 \cdot 5 & 68.5\end{array}$

$117 \quad 55$

$116.5 \quad 18.4$

$114 \quad 9 \cdot 3$

$110 \quad 1 \cdot 12$

$107 \quad 0.174$

$108 \quad 0.096$

$107 \quad 0.049$

$106 \quad 0.020$

$105.5 \quad 1.03$

103

$103 \cdot 5$

$\begin{array}{ll}100 & -\overline{123} * \\ 42 & 0 \cdot 123^{*} \\ 24 \cdot 8 & 0 \cdot 108 \\ 14 \cdot 2 & 0 \cdot 137 \\ 6 \cdot 25 & 0 \cdot 164 \\ 2.75 & 0.107 \\ 2 \cdot 14 & 0.081 \\ 1.22 & -\end{array}$

$\begin{array}{cc}100 & - \\ 48 & 0 \cdot 080^{*} \\ 37 \cdot 9 & 0 \cdot 092 \\ 12 \cdot 0 & 0 \cdot 125 \\ 5.95 & 0 \cdot 129 \\ 3.30 & 0 \cdot 129 \\ 1.64 & 0 \cdot 126 \\ 0.50 & 0 \cdot 110 \\ 0 \cdot 180 & 0 \cdot 117 \\ 0.048 & - \\ 1.15 & - \\ 5 \cdot 0 & - \\ 100 & -\end{array}$

$\begin{array}{cc}100 & - \\ 98 & - \\ 50 & 0.092^{*} \\ 33 \cdot 7 & 0.081 \\ 22 \cdot 2 & 0 \cdot 117 \\ 10.45 & 0.179 \\ 3.69 & 0.228 \\ 1.07 & 0.196 \\ 0.52 & -\end{array}$

$\begin{array}{ll}100 & - \\ 101 & - \\ 57 \cdot 5 & 0 \cdot 059^{*} \\ 47 & 0 \cdot 086 \\ 15 \cdot 8 & 0 \cdot 117 \\ 8 \cdot 15 & 0 \cdot 182 \\ 1 \cdot 02 & 0 \cdot 196 \\ 0 \cdot 162 & 0 \cdot 163 \\ 0 \cdot 089 & 0 \cdot 142 \\ 0 \cdot 039 & 0 \cdot 155 \\ 0 \cdot 019 & - \\ 0 \cdot 98 & - \\ 4 \cdot 1 & - \\ 100 & -\end{array}$

Series 37. 9.8,'28.

11.36 a.m. $55 \quad$ Weak Sun

L Photometer. At El. 11.45,

Sun and light clouds. 11.51 ", 56

Dark currents rather 11.55 ," ,

troublesome. High water 11.57 ,"

10.57 a.m.

NEW SERIES.-VOL, XVI. NO. 1. MAY, 1929. 
Date, Renarks, etc.

$\stackrel{\text { T }}{\text { G.M.T. }}$

$a$
55
$"$,
54
53
50
49
47
46
45
43
41
40

Series 39. 29.8.'28.

12.51 p.m. 55

Series 38. 9.8.'28.

Light.

K Photometer. Other 12.58, conditions as in Series 37. 1.4,

Secchi dise $10 \mathrm{~m}$. in 1.14 , shadow of ship and $9 \mathrm{~m}$. 1.29 ," in sun at 3.30 p.m. $\left(V_{\mathrm{a}} \quad 1.58\right.$, about 50,000 m.c.).

$\begin{array}{lll}2.10 & , & 4 \\ 2.32 & , & 47 \\ 2.36 & " & 46 \\ 2.40 & , & 45 \\ 2.54 & , & 43 \\ 3.12 & , & 41 \\ 3.22 & \end{array}$

Wind S.W., light. Con- 12.17 ",

siderable swell, some 12.22 ,"

waves breaking. Clear 12.26 ,

sky with light clouds. 12.32 ",

High water 4.38 p.m.

12.44,
12.50,

Series 40, 29.8.'28.

1.5 p.m. 48

K Photometer. Other

conditions as in Series 39.

Secchi disc $15 \mathrm{~m}$. on lee

and shadow side of ship,

but illuminated by sun.

light passing under the

keel, at 3.28 p.m., $14 \mathrm{~m}$.

on weather side ( $\mathrm{V}$ about

80,000 m.c.).

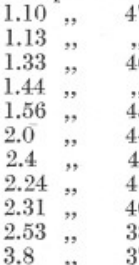

Sertes 4l. 18.9.'28.

1.45 p.m. 38

K Photometer. At EI, 1.51 , 37

Calm. Sky clear after

thick fog in morning.

light very steady. High

water 8.6 p.m. Secehi

disc $16.5 \mathrm{~m}$. in shadow of

ship and $14 \mathrm{~m}$. in sun at

4.30 p.m. ( $V$ about 25,000 m.c.).

1.55,

$1.59, \quad 36$

$2.25,, \quad 35$

(1)

$2.32, \quad 33$

2.35,

2.42 ",

2.46 , ,

3.0 ,

3.16,

3.24,

3.29,

3.35,

3.45,

3.54,

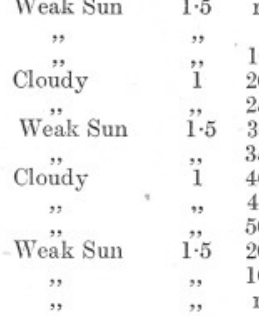

$\beta$

d $\quad \mathrm{V}_{\mathrm{a}}$

V

$\stackrel{\mathrm{p}}{\%}$

100

47
53
43
$28 \cdot 0$
$33 \cdot 2$
42
$41 \cdot 5$
$28 \cdot 8$
$27 \cdot 1$
$27 \cdot 6$
$38 \cdot 5$
$39 \cdot 2$
$52 \cdot 5$

47

18.

0.525

0.357

$0 \cdot 41$

$0 \cdot 259$

$0 \cdot 105$

$0 \cdot 054$

0.028

$1 \cdot 42$

$7 \cdot 65$

$52 \cdot 5$

Bright Sun

$108.5 \quad 108 \cdot 5$

$109 \cdot 5 \quad 52 \cdot 5$

$109 \cdot 5 \quad 26 \cdot 3$

$108 \quad 15.8$

109

108

$111 \cdot 5$

$9 \cdot 2$

3.04

$2 \cdot 64$

$109 \cdot 5$

1.56

Bright Sun

110

110

$97 \quad 46.5$

$\begin{array}{rl}97 \cdot 5 & 21 \cdot 1 \\ 106 \cdot 5 & 13 \cdot 15\end{array}$

$104 \cdot 5 \quad 3 \cdot 03$

$101 \quad 0.775$

$\begin{array}{ll}55.5 & 0.435\end{array}$

Light Cloud

56

Bright Sun

$0 \cdot 204$

$94 \quad 0.306$

$92.5 \quad 0.082$

,"

$0 \cdot 082$
2.42

$83 \cdot 5$

$34 \cdot 2$

$5 \cdot 5$

$1 \cdot 87$
$1 \cdot 07$

$0.975 \quad 0.054$

$0.625 \quad 0 \cdot 098$

$0.365 \quad 0.112$

$0.199 \quad 0.129$

$0 \cdot 100$

$3 \cdot 70$

$19 \cdot 6$

100

100

$48 \quad 0.126 *$

$24 \cdot 0 \quad 0.119$

$14 \cdot 6 \quad 0 \cdot 104$

$8.45 \quad 0.164$

$2.82 \quad 0.127$

$2.36 \quad 0.068$

$1 \cdot 4$

100

48

$21 \cdot 6$

$12 \cdot 3$

$0.765 \quad 0 \cdot 103$

0.785

$0 \cdot 365$

0.326

0.088

$2 \cdot 83$

$83 \cdot 5$

100

Sun

$78 \cdot 5 \quad 78 \cdot 5$

$77 \cdot 5 \quad 41 \cdot 5$

,"

,

,

",

$\begin{array}{ll} & 25 \\ & \end{array}$

Light Cloud $\quad 1.5 \quad 35$

$\begin{array}{lll}\text { Sun } & 2 & 40\end{array}$

$\begin{array}{lll}\text { Variable } \quad " & 45 \\ & & 25\end{array}$

Light Cloud

$27 \cdot 7$

$19 \cdot 2$

$11 \cdot 2$

$7 \cdot 05$

$4 \cdot 3$

$1 \cdot 84$

$1 \cdot 38$

$0 \cdot 78$

$4 \cdot 8$

$2 \cdot 79$

$0 \cdot 435$

$0 \cdot 116$

$0 \cdot 114$

$3 \cdot 25$

$29 \cdot 2$

100

$53 \cdot 5$

$36 \cdot 0$

$25 \cdot 0$

$15 \cdot 6$

$10 \cdot 0$

$6 \cdot 25$

$3 \cdot 68$

$2 \cdot 03$

$1 \cdot 13$

$7 \cdot 15$

$7 \cdot 8$

$1 \cdot 24$

$0 \cdot 357$

$0 \cdot 294$

$9 \cdot 9$

100

Series 42. 2.10''28.

1.10 p.m. 35

L Photometer. At El.

Very faint air from N.W.

Very slight roll. Sun shining through high hazy elouds. High water

7.34 p.m. Flat calm with

glassy water surface

later.

$1.19, \quad 34$

$1.36, \quad 33$

1.39,

$1.46, \quad 32$

$1.57, \quad 31$

$2 \cdot 3, \quad 30$

2.11 ,"

29

Series 43. 12.10.'28.

12.30 p.m. $\quad 39$

J Photometer. About 12.48,

$3 \mathrm{~m}$. S.W. of Mewstone.

$1.3 "$,

Wind N.E. Considerable

swell. White clouds

$1 \cdot 18, \quad 30$

moving rapidly across the

1.30,

sky. High water 5.5 p.m.

Secchi dise $6.5 \mathrm{~m}$. on

either side of ship at 1.40

p.m.

,"

",

,

,

,

,

Sun

Light Cloud $\quad$ औ.5 $\quad 40$

Variable

Cloudy

$0 \cdot 365$

0.445

$0 \cdot 56$

0.68

$1 \cdot 31$

$2 \cdot 39$

1.46

$2 \cdot 66$

$5 \cdot 25$

$9 \cdot 2$

$17 \cdot 7$

$4 \cdot 5$

$8 \cdot 9$

$16 \cdot 1$

$32 \cdot 2$

54

100

62

62

62

$0 \cdot 93$

$0 \cdot 013$

0.675

100

$1 \cdot 50$

$0 \cdot 026$

$30 \cdot 6$

$30 \cdot 6$

100

$0.086 *$

$0 \cdot 076$

$0 \cdot 084$

0.092

0.092

$0 \cdot 117$

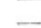

0.096

-

0.

0.126

$0 \cdot 123$

$0 \cdot 131$

$0 \cdot 128$

$0 \cdot 129$

$0 \cdot 166^{*}$ 


\begin{tabular}{|c|c|c|c|c|c|c|c|c|c|c|}
\hline Date, Remarks, etc. & $\begin{array}{r}\mathrm{T} \\
\text { G.M }\end{array}$ & I.T. & $a$ & Light. & $\beta$ & $\begin{array}{c}\mathrm{d} \\
\text { metres }\end{array}$ & $\begin{array}{c}V_{\mathrm{a}} \\
\text { k.m.c. }\end{array}$ & $\begin{array}{c}\text { V } \\
\text { k.m.c. }\end{array}$ & $\begin{array}{l}\mathrm{p} \\
\mathrm{\%}\end{array}$ & $\lambda$ \\
\hline $\begin{array}{l}\text { SERIES } 44 . \text { 30.11.'28. } \\
\text { J Photometer. At El. } \\
\text { Wind N.W., fresh. } \\
\text { Waves breaking. Moder- } \\
\text { ate short swell. Sky com- } \\
\text { pletely overcast. High } \\
\text { water } 7.51 \text { p.m. Secchi } \\
\text { disc } 11 \mathrm{~m} \text {. to windward, } \\
9 \mathrm{~m} \text {. to leeward at } 3 \text { p.m. }\end{array}$ & $\begin{array}{l}12.37 \mathrm{p} \\
12.52 \\
12.56 \\
12.58 \\
1.1 \\
1.4 \\
1.8 \\
1.11 \\
1.22 \\
1.40 \\
1.50 \\
1.56 \\
2.1 \\
2.14 \\
2.34 \\
2.48\end{array}$ & 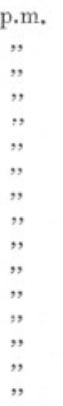 & $\begin{array}{l}- \\
- \\
- \\
- \\
- \\
- \\
- \\
- \\
- \\
- \\
-\end{array}$ & $\begin{array}{c}\text { Cloudy } \\
\text {," } \\
, \\
\text {," } \\
\text {," } \\
, \\
, \\
, \\
, \\
, \\
\text {, } \\
, \\
, \\
,\end{array}$ & $\begin{array}{l}1 \\
,, \\
, \\
", \\
, \\
, \\
, \\
", \\
, \\
, \\
, \\
, \\
", \\
,\end{array}$ & $\begin{array}{r}\mathrm{r} \\
5 \\
10 \\
15 \\
20 \\
25 \\
30 \\
35 \\
15 \\
35 \\
40 \\
45 \\
50 \\
55 \\
25 \\
\mathrm{r}\end{array}$ & $\begin{array}{l}15 \cdot 35 \\
8 \cdot 9 \\
7 \cdot 8 \\
8 \cdot 45 \\
7 \cdot 05 \\
6 \cdot 5 \\
7 \cdot 25 \\
7 \cdot 45 \\
6 \cdot 4 \\
6 \cdot 3 \\
7 \cdot 6 \\
6 \cdot 4 \\
5 \cdot 3 \\
5 \cdot 75 \\
6 \cdot 65 \\
5 \cdot 1\end{array}$ & $\begin{array}{l}15 \cdot 35 \\
3 \cdot 04 \\
1 \cdot 38 \\
0 \cdot 83 \\
0 \cdot 387 \\
0 \cdot 226 \\
0 \cdot 146 \\
0 \cdot 080 \\
0 \cdot 43 \\
0 \cdot 53 \\
0 \cdot 030 \\
0 \cdot 013 \\
0 \cdot 006 \\
0 \cdot 005 \\
0 \cdot 170 \\
5 \cdot 1\end{array}$ & $\begin{array}{l}100 \\
34 \cdot 2 \\
17 \cdot 7 \\
9 \cdot 8 \\
5 \cdot 5 \\
3 \cdot 48 \\
2 \cdot 01 \\
1 \cdot 07 \\
6 \cdot 7 \\
0 \cdot 84 \\
0 \cdot 395 \\
0 \cdot 205 \\
0 \cdot 115 \\
0 \cdot 085 \\
2 \cdot 56 \\
100\end{array}$ & $\begin{array}{c}-\overline{156} \\
0 \cdot 125 \\
0 \cdot 117 \\
0 \cdot 103 \\
0 \cdot 100 \\
0.117 \\
- \\
- \\
- \\
0 \cdot 141 \\
0 \cdot 123 \\
0 \cdot 082 \\
- \\
-\end{array}$ \\
\hline $\begin{array}{l}\text { SERIEs 45. 2.1.'29. } \\
\text { L Photometer. At El. } \\
\text { Strong N.E. wind. } \\
\text { Waves breaking every- } \\
\text { where. Very heavy swell. } \\
\text { Cloudy sky. Light very } \\
\text { variable. High water } \\
11.5 \text { p.m. }\end{array}$ & $\begin{array}{l}12.35 \mathrm{p} \\
12.45 \\
12.52 \\
12.59\end{array}$ & $\begin{array}{l}\text { p.m. } \\
, \\
, \\
,\end{array}$ & $\begin{array}{l}- \\
- \\
-\end{array}$ & $\begin{array}{c}\text { Cloudy } \\
\text {," } \\
\text { ", } \\
\text {, }\end{array}$ & $\begin{array}{l}1 \\
,, \\
,\end{array}$ & $\begin{array}{r}5 \\
10 \\
15 \\
20\end{array}$ & $\begin{array}{l}15 \cdot 0 \\
12 \cdot 45 \\
13 \cdot 7 \\
15 \cdot 25\end{array}$ & $\begin{array}{l}6 \cdot 3 \\
2 \cdot 98 \\
2 \cdot 10 \\
1 \cdot 39\end{array}$ & $\begin{array}{r}42 \\
23 \cdot 9 \\
15 \cdot 3 \\
9 \cdot 1\end{array}$ & $\begin{array}{l}0 \cdot 127^{*} \\
0 \cdot 101 \\
0 \cdot 097 \\
-\end{array}$ \\
\hline $\begin{array}{l}\text { SERIES 46. 2.1.'29. } \\
\text { J Photometer. Other } \\
\text { conditions as in Series } 45 \text {. }\end{array}$ & $\begin{array}{l}1.27 \mathrm{p} \\
1.34 \\
1.47 \\
1.52 \\
1.54\end{array}$ & $\begin{array}{l}\text { p.m. } \\
\text {," } \\
, " \\
,, \\
,,\end{array}$ & $\begin{array}{l}- \\
- \\
- \\
-\end{array}$ & $\begin{array}{c}\text { Cloudy } \\
\text {," } \\
, " \\
,, \\
,,\end{array}$ & $\begin{array}{l}1 \\
, \\
, \\
, \\
,\end{array}$ & $\begin{array}{r}\mathrm{r} \\
5 \\
15 \\
25 \\
35\end{array}$ & $\begin{array}{l}7 \cdot 15 \\
7 \cdot 65 \\
9 \cdot 4 \\
7 \cdot 45 \\
7 \cdot 5\end{array}$ & $\begin{array}{l}7 \cdot 15 \\
2 \cdot 66 \\
1 \cdot 01 \\
0 \cdot 315 \\
0 \cdot 156\end{array}$ & $\begin{array}{c}100 \\
34 \cdot 8 \\
10 \cdot 7 \\
4 \cdot 25 \\
2 \cdot 08\end{array}$ & $\begin{array}{l}-\overline{138} \\
0 \cdot 13{ }^{*} \\
0 \cdot 106 \\
0 \cdot 082 \\
-\end{array}$ \\
\hline
\end{tabular}

Series 28, obtained on April 19th, is remarkable for the high value of $\mathrm{V}_{\mathrm{a}}$, i.e. 134,000 m.c., obtained at $12.56 \mathrm{p} . \mathrm{m}$. with a solar altitude of $51^{\circ}$. This value was the highest but one obtained during the year, and is probably attributable to the combination of clear sun and white clouds close to it. The value found for $\beta$ at 12.4 p.m. was $3 \cdot 75$. We may compare this with the vertical illumination, 126,000 m.c., found in Series 32 on July 4th at $12.52 \mathrm{p} . \mathrm{m}$. with $\alpha=62^{\circ}$. The only clouds in the sky on this occasion were low down, and the diffuse light was remarkably low, the value found for $\beta$ being over 6 , which is quite exceptional. This would give 105,000 m.c. as the pure sunlight in $V_{a}$ on July 4th, as compared with 98,000 m.c. on April 19th.

The highest value recorded for $\mathrm{V}_{\mathrm{a}}$ during the year was 139,500 m.c. at 12.39 p.m. on July 11th, $\alpha$ being $62^{\circ}$ (Series 33). On this occasion, also, the diffuse light was increased by the reflection from white clouds, as is shown by the fact that a measurement of $\beta$ at 12.36 p.m. gave a value as low as $3 \cdot 14$ with $\mathrm{V}_{\mathrm{a}}=137,000$ m.c. This would mean that at 12.39 p.m. the direct sunlight amounted to about 95,000 m.c., and the diffuse light to about 44,500 m.c.

Series 28 is also interesting as showing the small effect of sudden changes of surface light on the percentage illumination at any depth. An examination of the readings from 12.31 to 12.50 p.m. and from 3.53 to 4.1 p.m. shows that the passage of a cloud across the sun reduces 
the aerial and submarine illuminations in about the same ratio. This accords with all our previous experience that the vertical absorption coefficient of the sea is but little affected by the obliquity of the incident light. It would seem, as already suggested, that the surface and surface layers generally have sufficient diffusing properties to control the average obliquity of the light in the deeper layers.

TABLE II.

Station E1, Collected Results.

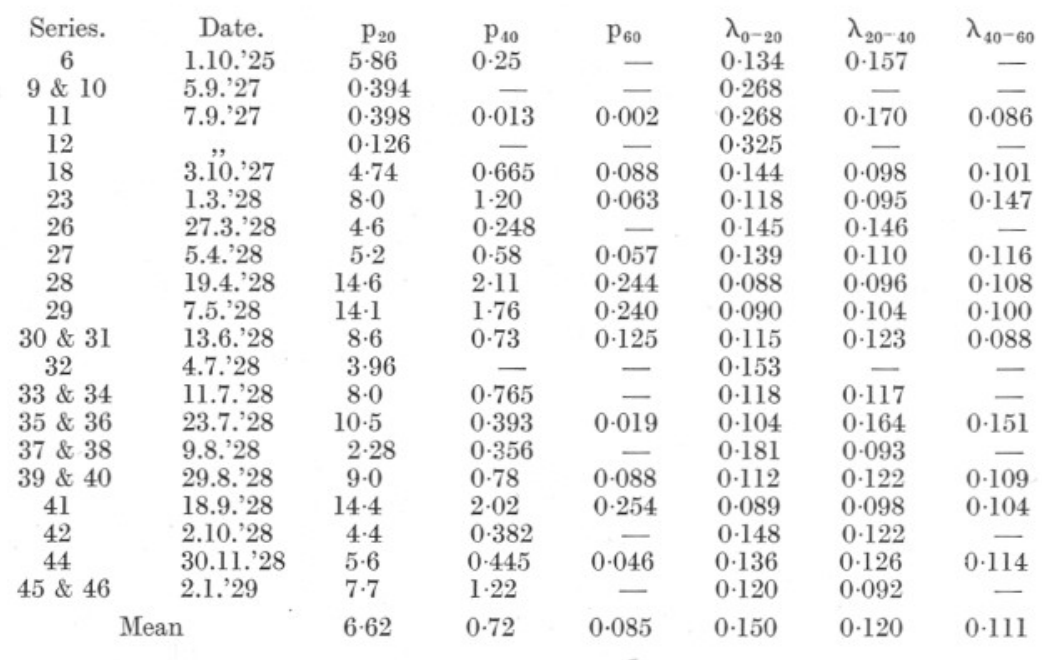

Table II shows the percentage illuminations at 20,40, and 60 metres, respectively, for all the series obtained so far at E1, and also the values of the mean absorption coefficients for the intervals 0 to 20,20 to 40 , and 40 to 60 metres. All series obtained closer in-shore have been omitted from this table, which is an attempt to show the seasonal changes in opacity.

When two series were taken on one day their results have been combined in the table, except in the case of Series 11 and 12. These were obtained in the autumn of 1927 , when the water was very opaque, and such a large increase occurred in the opacity of the surface layers between the two series that the results differed entirely, and are shown separately. The percentage recorded at any depth is the mean of all values obtained at that depth or calculated for it from values obtained for levels within $5 \mathrm{~m}$. above or below. In the single case of Series 30 and 31 , as no readings were taken in Series 31 between $10 \mathrm{~m}$. and $30 \mathrm{~m}$., the geometric mean of the percentages at these levels was used, and 
combined with the $20 \mathrm{~m}$. percentage of Series 30 to obtain the value given.

The values of $\lambda$, which measures the opacity of the water, are calculated from the mean percentages. A mean surface loss of $15 \%$ is assumed in finding $\lambda_{0-20}$.

$$
\text { Hence, since } \frac{\log _{\mathrm{e}} 10}{20}=0 \cdot 115, \lambda_{0-20}=0 \cdot 115\left(\log 85-\log \mathrm{p}_{20}\right), \lambda_{20-40}=
$$

$0 \cdot 115\left(\log \mathrm{p}_{20}-\log \mathrm{p}_{40}\right)$, and $\lambda_{40-60}=0 \cdot 115\left(\log \mathrm{p}_{40}-\log \mathrm{p}_{60}\right)$.

In finding the mean values of $\mathrm{p}$ given in the last line allowance has been made for the fact that occasionally, when the surface water was rather opaque, so that $\mathrm{p}_{20}$ was low, there was not sufficient light to measure at $60 \mathrm{~m}$. and $\mathrm{p}_{60}$ is missing. Sometimes even $\mathrm{p}_{40}$ is absent. Thus, the simple averages of the figures given would exaggerate the percentages for the lower levels by eliminating the values corresponding to high opacity.

Accordingly, the average values of $\mathrm{p}_{20}$ were found, (a) for all the series, (b) for series containing values of $\mathrm{p}_{40}$, and (c) for series containing values of $\mathrm{p}_{60}$. The three means were $6 \cdot 62,7 \cdot 53$, and $8 \cdot 65$, respectively. The average of the values of $p_{40}$ was, accordingly, multiplied by $6 \cdot 62 / 7 \cdot 53$, and that for $\mathrm{p}_{60}$ by $6 \cdot 62 / 8 \cdot 65$. This will give a fair representation of the mean percentages if the average opacity of the lower layers is independent of that of the surface layer. This assumption seems to be borne out by the readings, as we sometimes find very clear water underlying very opaque water (Series 11), while occasionally the effect is reversed (Series 35 and 36).

The mean values of the absorption coefficients are the simple averages of the figures in the columns, since, as we have seen above, there is no reason to suppose that a high opacity in the upper layer would correspond to a high opacity lower down. It must be noted that the mean opacities found in this way, though shown in the same line as the mean values of $p$, differ appreciably from the opacities that would be found by calculation from the mean percentages, since the latter are the arithmetic, and not the geometric means of the individual values.

\section{Visibility of the Secchi Disc.}

Table III shows a comparison of the maximum depth of visibility of the Secchi disc with other data from Series 23 to 44 . The values of $\mathrm{V}_{\mathrm{a}}$ are, in some cases, estimates from photometer readings taken a little time before or after. D is the maximum depth of visibility on whichever side of the ship gave the greatest value. The percentage illumination $\mathrm{P}$ at the depth $\mathrm{D}$ is found from the value of the product $\mathrm{D} \lambda_{0-20}$ assuming a surface loss of $15 \%$. 
It will be seen that $\mathrm{D} \lambda_{0-20}$ varies less than either $\mathrm{D}$ or $\lambda_{0-20}$, its mean value being $1 \cdot 70$. Hence, a very rough rule for finding the opacity is $\lambda_{0-20}=1 \cdot 7 / \mathrm{D}$. Another way of expressing the same statement is that the percentage illumination at the limit of visibility is about $16 \%$.

There seems to be no relation between $\mathrm{D}$ and either $\beta$ or $\mathrm{V}_{\mathrm{a}}$. $\mathrm{V}$, the illumination at the limit of visibility, varies between 800 and 18,700 m.c. This is entirely in accord with some previous observations, which showed that the visibility of the disc was not affected by the approach of nightfall until the light became so poor that the adaptive power of the eye could no longer compensate for the reduction.

\section{TABLE III.}

\section{Visibility of the Secchi Disc.}

\begin{tabular}{|c|c|c|c|c|c|c|c|c|c|}
\hline & Date & Time & $\beta$ & $V_{a}$ & D & $\lambda_{0-20}$ & $\mathrm{D} \times$ & $\mathrm{P}$ & V \\
\hline Series. & 1928 & $\begin{array}{c}\text { G.M.T. } \\
\text { p.m. }\end{array}$ & & k.m.c. & $\mathrm{m}$. & & $\lambda_{0-20}$ & & k.m.c. \\
\hline 23 & 1,3 & 4.30 & 2 & 25 & 14 & $0 \cdot 118$ & $1 \cdot 65$ & $16 \cdot 2$ & $4 \cdot 0$ \\
\hline 24 & 6,3 & 2.40 & $2 \cdot 5$ & 60 & $13 \cdot 5$ & $0 \cdot 151$ & $2 \cdot 04$ & $11 \cdot 0$ & $6 \cdot 6$ \\
\hline 27 & $5,4=$ & 4.15 & 2 & 55 & 10 & $0 \cdot 139$ & $1 \cdot 39$ & $21 \cdot 2$ & $11 \cdot 6$ \\
\hline 28 & 19,4 & 4.18 & 3 & 65 & 18 & 0.088 & 1.58 & $17 \cdot 4$ & $11 \cdot 3$ \\
\hline 29 & 7,5 & 4.5 & 1 & $18 \cdot 7$ & 22 & $0 \cdot 090$ & 1.98 & $11 \cdot 7$ & $2 \cdot 2$ \\
\hline $30 \& 31$ & 13,6 & 3.17 & 1 & 40 & 14 & 0.115 & $1 \cdot 61$ & $15 \cdot 9$ & $6 \cdot 4$ \\
\hline 32 & 4,7 & 12.14 & 6 & 128 & $11 \cdot 5$ & $0 \cdot 153$ & $1 \cdot 76$ & $14 \cdot 6$ & $18 \cdot 7$ \\
\hline $33 \& 34$ & 11,7 & 3.28 & 2 & 87 & $18 \cdot 5$ & $0 \cdot 118$ & $2 \cdot 18$ & $9 \cdot 6$ & $8 \cdot 4$ \\
\hline $35 \& 36$ & 23,7 & $3 \cdot 26$ & 3 & 100 & $17 \cdot 5$ & $0 \cdot 104$ & $1 \cdot 82$ & $13 \cdot 7$ & $13 \cdot 7$ \\
\hline $37 \& 38$ & 9,8 & $3 \cdot 30$ & $1 \cdot 5$ & 50 & 10 & $0 \cdot 181$ & 1.81 & $13 \cdot 9$ & $7 \cdot 0$ \\
\hline $39 \& 40$ & 29,8 & $3 \cdot 28$ & 3 & 80 & 15 & 0.112 & $1 \cdot 68$ & $15 \cdot 8$ & $12 \cdot 6$ \\
\hline 41 & 18,9 & 4.30 & 1.5 & 25 & $16 \cdot 5$ & 0.089 & $1 \cdot 47$ & $19 \cdot 5$ & $4 \cdot 9$ \\
\hline 43 & 12,10 & 1.40 & 1 & 30 & $6 \cdot 5$ & $0 \cdot 203$ & $1 \cdot 32$ & $22 \cdot 6$ & $6 \cdot 8$ \\
\hline \multirow[t]{2}{*}{44} & $30,1]$ & 3.0 & 1 & $4 \cdot 5$ & 11 & $0 \cdot 136$ & $1 \cdot 50$ & 18.8 & $0 \cdot 8$ \\
\hline & & & & & Mean & & $1 \cdot 70$ & $15 \cdot 8$ & \\
\hline
\end{tabular}

It would seem, then, that we may use the Secchi disc to estimate the opacity of the water without paying any attention to even comparatively large variations in the brightness of the daylight. On the other hand, the large variations recorded in the penultimate column of Table III show that other sources of error, which must always be large where a visual estimate has to be made under very varying conditions, prevent the method from being anything more than a rough approximation.

In the 1928 paper we mentioned the desirability of trying a water telescope through which to view the Secchi disc. With any form of rigid tube it would be quite impossible to keep the disc in view when the ship was rolling more or less heavily, as was almost always the case. Accordingly attempts were made to use a wide cloth tube, about 0.3 metre in diameter and $2 \mathrm{~m}$. in length. The attempts were, however, quite unsuccessful. Trials were also made with a Nicol prism, which under certain conditions extinguishes reflections from water, but with the 
very broken water surfaces usually encountered there was a positive disadvantage in its use. We were no more successful with various colour screens, though certain of these were often of use in picking up objects on the shore in hazy weather. The depths recorded for the visibility limit of the disc are those obtained by the youngest of the deck hands, who has remarkably good sight and could often see the disc half a metre or a metre further down than we could.

\section{Reliability of Data.}

Before discussing the interpretation of the results, it is necessary to consider what differences in the value of $\lambda$ are significant. It was concluded in the previous work (1928) that the two photometers, $\mathrm{L}$ (vacuum) and $\mathrm{K}$ (gas-filled) gave results which were generally in agreement. In the course of the present work, $\mathrm{K}$ and $\mathrm{L}$ were compared very thoroughly, and were found to be in close agreement at times, so that one can rely on the differences, when found, as being of definite significance. Table IV contains data from Series 21 (see 3, p. 474), obtained with photometer K, and shows the consistency of the results obtained under certain conditions. Furthermore, the altitude of the sun appears to have but little influence upon the value of $\lambda$ though a slight rise occurred as the morning advanced and the altitude increased. On this occasion $\beta$ was approximately 2, so that about half of the total light was direct sunlight.

\section{TABLE IV.}

From Series 21, Рhotometer K.

$\begin{array}{rccc}\text { Hour. } & a & \mathrm{~d} & \lambda \\ 11.22 & 24^{\circ} & 0-10 & 0 \cdot 160 \\ 9.36 & 26^{\circ} & 0-20 & 0 \cdot 130 \\ 10.21 & 30^{\circ} & 0-20 & 0 \cdot 134 \\ 11.50 & 35^{\circ} & 0-20 & 0 \cdot 137 \\ 9.22 & 24^{\circ} & 0-40 & 0 \cdot 121 \\ 10.28 & 31^{\circ} & 0-40 & 0 \cdot 121 \\ 12.6 & 35^{\circ} & 0-40 & 0 \cdot 124\end{array}$

Table $\mathrm{V}$ records values of $\lambda$ obtained with the two photometers. The results agree closely at 10,15 , and 20 metres. There is an indication that the more opaque layers are found at lesser depths as the day goes on. The differences in the values of $\lambda$ are quite unconnected with any slight differences in salinity, nor does there appear to be any relation between the value of $\lambda$ and the temperature of the water. This point will be mentioned again later. 
The values of $\lambda$ for the layer next the surface is affected by the surface loss which is somewhat variable. The unsteadiness of the light just below the surface renders any method of measurement involving a telephone balance unsuitable for measuring this loss accurately, save under exceptionally favourable conditions. Moreover, a diffusing window is absolutely essential for this purpose, as the obliquity correction for the different conditions above and below the surface with the groundglass window used in Series 7 to 23 , inclusive, was too uncertain to justify any importance being attached to measurements of surface loss.

\section{TABLE V.}

Station E1, Series 9, 12.14-1.13 G.M.T. downwards.

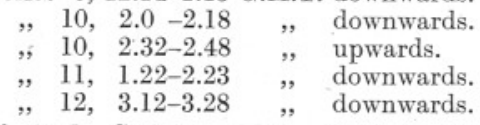

Series 9 and $10, \beta=1$. Series $11, \beta=4$. Series $12, \beta=3$. Series 9 and 10 on 5.9.'27.

$\begin{array}{cccccccc}\mathrm{d} & \lambda(\mathrm{K}) & \lambda(\mathrm{L}) & \lambda(\mathrm{L}) & \mathrm{t}^{\circ} & \mathrm{S} \% & \lambda(\mathrm{K}) & \lambda(\mathrm{L}) \\ 0 & - & - & - & 16 \cdot 1 & 35 \cdot 13 & - & - \\ 2 \cdot 5 & - & 0 \cdot 349^{*} & 0 \cdot 356^{*} & - & - & 0 \cdot 318^{*} & 0 \cdot 523^{*} \\ 5 & 0 \cdot 350^{*} & 0 \cdot 318 & 0 \cdot 294 & - & - & 0 \cdot 308^{*} & 0 \cdot 387 \\ 10 & 0 \cdot 268 & 0 \cdot 266 & 0 \cdot 232 & 15 \cdot 82 & 35 \cdot 16 & 0 \cdot 293 & 0 \cdot 306 \\ 15 & 0 \cdot 215 & 0 \cdot 212 & 0 \cdot 217 & 15 \cdot 76 & 35 \cdot 12 & 0 \cdot 267 & 0 \cdot 208 \\ 20 & 0 \cdot 151 & 0 \cdot 168 & 0 \cdot 170 & 15 \cdot 10 & 35 \cdot 17 & 0 \cdot 206 & 0 \cdot 175 \\ 25 & 0 \cdot 106 & - & - & 13 \cdot 80 & 35 \cdot 19 & 0 \cdot 179 & - \\ 30 & - & - & - & - & - & 0 \cdot 148 & - \\ 35 & - & - & - & - & - & 0 \cdot 128 & - \\ 40 & -- & - & - & - & - & 0 \cdot 128 & - \\ 45 & - & - & - & -- & - & 0 \cdot 082 & - \\ 50 & -- & - & - & 13 \cdot 36 & 35 \cdot 20 & 0 \cdot 051 & - \\ 55 & -- & - & - & - & - & 0 \cdot 061 & - \\ 70 & -- & - & - & 13 \cdot 36 & 35 \cdot 20 & - & -\end{array}$

The temperature and salinity measurements refer to September 7 th, but must be close approximations for September 5th also. The letters in brackets indicate the photometer, K or L, used.

Pending the results of further measurements of surface loss, which we hope to make by means of the time-integrating Neon Lamp Photometer devised by Dr. J. H. J. Poole (4), we have, throughout, assumed a surface loss of $15 \%$ as the mean of the results obtained with a diffusing window in Series 1 to 6 . The largest surface loss recorded in these series was $31 \%$ (Series 4 ) and the smallest $5 \%$ (Series 6 ).

It is evident that this assumption of a uniform loss will give us too small values of $\lambda$ for the upper layer when the surface is smooth and the 
surface loss small, and too high values for rough conditions, when the surface loss is large. The differences, however, are not very important. Remembering that $\lambda=\frac{2 \cdot 3}{10}\left(\log \mathrm{p}_{0}-\log \mathrm{p}_{10}\right)$, where $\lambda$ refers to a layer 10 metres thick, $p_{0}$ and $p_{10}$ being the percentage illuminations just below the surface and at a depth of 10 metres, respectively, we readily find that the use of $85 \%$ instead of $95 \%$, as the value of $\mathrm{p}_{0}$ on a very calm day, would reduce $\lambda$ by 0.011 . Similarly if the surface loss were as high as $31 \% \lambda$, as calculated, would be 0.021 too high.

These errors would not be of very great importance compared with the variations observed for $\lambda$. Moreover, for the values of $\lambda$ given in Table II for layers $20 \mathrm{~m}$. thick the errors for the upper layer would be only 0.005 and $0 \cdot 010$, respectively.

It is probable that errors due to the shadow of the ship are at least as important as those due to variations in the surface conditions.

Again, in deep water, the readings, though usually very steady, are small, and, consequently, even minute dark currents become highly important. Their presence, however, was carefully checked by closing the photometer window at each depth. The earlier series were also subject to a small error from soakage effects. These were eliminated by leaving the high tension plugged in continuously, and in the new arrangement, shown in the figure, this permanent connection of the high tension has been protected from any accidental displacement.

Bearing in mind these possible sources of error, one may arrive at an idea of the reliability of the various measurements, yet it is scarcely possible to assign a definite percentage value to the error, as the external conditions vary so much when the sea is rough. In particular, an error may arise through a small wave increasing the height of the column of water above the photometer, though unable to raise the ship bodily or, alternatively, the motion of the ship may raise or lower the photometer, thus rendering the light unsteady and hard to measure accurately. With the photometer at 10 metres, namely, in determining the value of $\lambda$ for 5 metres, it is unlikely that the depth error ever exceeds 1 metre; 0.5 metre seems, however, quite a possible value. Again, the shading due to the ship becomes relatively more important on a dull day than on a sunny day, when the greater proportion of the illumination is due to direct sunlight if due care is taken to keep the photometer out of the direct shadow.

The error due to the cable suspending the photometer not being perpendicular when the ship is drifting, has already been shown to be negligible (1926, p. 189).

To sum up, it is probably safe to conclude that with photometers $\mathrm{K}$ and $\mathrm{J}$ between 10 and 30 metres or with photometer $\mathrm{L}$ between 10 and 
25 metres, a $10 \%$ error in $\lambda$ is unlikely, a $5 \%$ error is unusual, and the results are often considerably more accurate.

\section{Causes of the Observed Significant Differences in the Values of the Absorption Coefficient.}

In our 1928 paper, we suggested that the variation in the values of $\lambda$ was due to the different horizontal distribution of the phytoplankton and to the zooplankton ascending or descending according to the position of the optimum light intensity for each species. We attributed the much greater clearness of the water in winter as compared with September to the fact that the sea is far poorer in plankton in mid-winter than in autumn. The high opacity of the surface layers which occurred in the autumn of 1927, and declined with the approach of winter, was not repeated in 1928, nor was it found off-shore in 1925, so the conditions in 1927 appear to have been somewhat abnormal. There is, of course, a certain absorption of light, due to inorganic matter in suspension, at all times of the year, for the whole of the English Channel is subject to detrital sedimentation. The present paper contains the results of an examination of the water throughout an entire year. We have, in addition, the data from September to December, 1927. It may be said at once that no single factor appears to be responsible for the variation in the values of $\lambda$ throughout the year and throughout the day. As possible causes, we have to consider the inorganic material in suspension, the phytoplankton, the zooplankton, and the organic débris resulting from the living organisms or derived from the land or the bottom. Since the phytoplankton are developed near the surface in those layers in which the illumination is adequate, and since in summer the upper 15 to 25 metres is also warmer, one might expect to find some correlation between the temperature of the water and the value of $\lambda$. Table VI shows the temperatures and the values of $\lambda$ obtained with the different photometers at Station E1. It should be added that Series 22 of December 14th, 1927, relates to a position near the Eddystone Lighthouse, which is 10 miles nearer shore than is Station E1. It has been inserted on account of its being the only measurement available for that winter, and the water further out to sea must have been at least as clear as at the Eddystone. Inspection of the data in Table VI reveals no such supposed correlation between high values of $\lambda$ and the warm surface layer, save in September, 1927. In July, 1928, however, the clearest water was found near the surface, consequently, the view that the phytoplankton is a really important factor in causing the absorption of light in the sea must be ruled out, though it would be premature to conclude that it is of no importance. 
TABLE VI.

\begin{tabular}{|c|c|c|c|c|c|c|c|c|c|c|c|c|c|c|c|}
\hline Ser. & $1 / 10$ & ${ }^{6}$ & $\begin{array}{l}{ }^{9} \\
\quad 5 / 9\end{array}$ & $27^{10}$ & & $\begin{array}{c}11 \\
7 / 9 / ' 27\end{array}$ & & $3 / 10$ & $\begin{array}{l}18 \\
/ 27\end{array}$ & $14 / 1$ & $\begin{array}{l}22 \\
2 / 27\end{array}$ & & $28^{23}$ & $27 /$ & / 28 \\
\hline $\mathrm{m}$. & $\mathrm{t}^{\circ} \mathrm{C}$ & $\lambda(\mathrm{K})$ & $\lambda(\mathrm{K})$ & $\lambda(\mathrm{L})$ & $\mathrm{t}^{\circ} \mathrm{C}$ & $\lambda(\mathrm{K})$ & $\lambda(\mathrm{L})$ & $\mathrm{t}^{\circ} \mathrm{C}$ & $\lambda(\mathrm{K})$ & $\mathrm{t}^{\circ} \mathrm{C}$ & $\lambda(\mathrm{K})$ & $\mathrm{t}^{\circ} \mathrm{C}$. & $\lambda(\mathrm{K})$ & $\mathrm{t}^{\circ} \mathrm{C}$ & $\lambda(\mathrm{K})$ \\
\hline 0 & $14 \cdot 9$ & - & - & - & $16 \cdot 1$ & - & - & $13 \cdot 9$ & - & $11 \cdot 8$ & - & $9 \cdot 7$ & - & $9 \cdot 6$ & \\
\hline $2 \cdot 5$ & - & - & - & $0 \cdot 349^{*}$ & - & $0 \cdot 318^{*}$ & $0.523^{*}$ & - & - & - & - & - & - & & \\
\hline 5 & $14 \cdot 50$ & $0 \cdot 134$ & $0.350^{*}$ & $0 \cdot 318^{*}$ & - & $0 \cdot 308^{*}$ & $0 \cdot 387$ & $13 \cdot 80$ & $0 \cdot 166^{*}$ & $11 \cdot 8$ & $0 \cdot 128$ & $9 \cdot 45$ & $0 \cdot 127^{*}$ & $9 \cdot 62$ & $0.172^{*}$ \\
\hline 10 & $14 \cdot 00$ & - & 0.268 & $0 \cdot 266$ & $15 \cdot 82$ & $0 \cdot 293$ & $0 \cdot 306$ & - & 0.099 & - & $0 \cdot 110$ & $9 \cdot 32$ & $0 \cdot 103$ & - & $0 \cdot i 26$ \\
\hline 15 & $13 \cdot 83$ & $0 \cdot 143$ & $0 \cdot 215$ & $0 \cdot 212$ & $15 \cdot 76$ & $0 \cdot 267$ & $0 \cdot 208$ & $13 \cdot 80$ & $0 \cdot 115$ & - & -- & - & 0.099 & - & $0 \cdot 117$ \\
\hline 20 & $13 \cdot 70$ & - & $0 \cdot 151$ & $0 \cdot 168$ & $15 \cdot 10$ & $0 \cdot 206$ & $0 \cdot 175$ & - & $0 \cdot 149$ & $11 \cdot 8$ & $0 \cdot 093$ & - & $0 \cdot 103$ & $9 \cdot 60$ & $0 \cdot 145$ \\
\hline 25 & - & $0 \cdot 162$ & $0 \cdot 106$ & 一 & $13 \cdot 83$ & $0 \cdot 179$ & - & $13 \cdot 76$ & $0 \cdot 121$ & - & - & $9 \cdot 32$ & $0 \cdot 101$ & - & $0 \cdot 162$ \\
\hline 30 & $13 \cdot 60$ & - & - & - & - & $0 \cdot 148$ & - & - & 0.063 & - & $0 \cdot 077$ & - & 0.094 & - & $0 \cdot 160$ \\
\hline 35 & - & - & - & - & - & $0 \cdot 128$ & - & - & 0.080 & - & - & - & $0 \cdot 101$ & - & $0 \cdot 127$ \\
\hline 40 & $13 \cdot 58$ & - & - & - & - & $0 \cdot 128$ & - & - & 0.089 & - & - & - & $0 \cdot 109$ & - & - \\
\hline 45 & - & - & - & - & - & 0.082 & - & - & $0 \cdot 127$ & $11 \cdot 8$ & - & - & $0 \cdot 127$ & - & - \\
\hline 50 & $13 \cdot 59$ & - & - & - & $13 \cdot 36$ & 0.051 & - & $13 \cdot 71$ & $0 \cdot 108$ & - & - & - & 0.138 & - & - \\
\hline 55 & - & - & - & - & - & 0.061 & - & - & $0 \cdot 068$ & - & - & - & $0 \cdot 156$ & - & - \\
\hline 60 & - & - & - & - & - & - & - & - & $0 \cdot 129$ & - & - & - & $0 \cdot 198$ & - & - \\
\hline 65 & - & - & - & - & - & - & - & - & & - & - & - & $0 \cdot 144$ & - & - \\
\hline 70 & $13 \cdot 57$ & - & - & - & $13 \cdot 36$ & - & - & $13 \cdot 70$ & & - & - & $9 \cdot 19$ & - & $9 \cdot 60$ & - \\
\hline
\end{tabular}


TABLE VI-(continued).

\begin{tabular}{|c|c|c|c|c|c|c|c|c|c|c|c|c|c|c|c|c|c|}
\hline $\begin{array}{l}\text { Ser. } \\
\text { m. }\end{array}$ & $\begin{array}{l}5 / 4 \\
\mathrm{t}^{\circ} \mathrm{C} .\end{array}$ & $\begin{array}{c}27 \\
\lambda(\mathrm{K}) \\
\lambda(\mathrm{K})\end{array}$ & $\begin{array}{l}19 / 4 \\
t^{\circ} \mathrm{C} .\end{array}$ & $\begin{array}{c}28 \\
1 / 28 \\
\lambda(\mathrm{K})\end{array}$ & $\mathrm{t}^{\circ} \mathrm{C}^{7 / 5 /}$ & $\begin{array}{l}29 \\
\lambda(\mathrm{K})\end{array}$ & $\mathrm{t}^{\circ} \mathrm{C}$ & $\begin{array}{c}30 \\
13 / 6 / 28 \\
\lambda(\mathrm{L})\end{array}$ & $\begin{array}{c}31 \\
\lambda(\mathrm{K})\end{array}$ & $\begin{array}{l}4 / 77^{\prime} \\
t^{\circ} \mathrm{C} \text {. }\end{array}$ & $\begin{array}{l}32 \\
28 \\
\lambda(\mathrm{L})\end{array}$ & $\mathrm{t}^{\circ} \mathrm{C}$ & $\begin{array}{c}33 \\
11 / 7 / 28 \\
\lambda(\mathrm{L})\end{array}$ & $\begin{array}{c}34 \\
\lambda(\mathrm{K})\end{array}$ & C. & $\begin{array}{c}35 \\
23 / 7 /{ }^{\prime} \\
\lambda(\mathrm{L})\end{array}$ & $\begin{array}{r}28{ }^{36} \\
\lambda(\mathrm{K})\end{array}$ \\
\hline 0 & - & -- & $10 \cdot 1$ & - & 12.5 & - & $13 \cdot 1$ & - & - & $14 \cdot 1$ & & $16 \cdot 1$ & - & - & $18 \cdot 3$ & - & - \\
\hline 2.5 & - & - & - & - & - & - & - & - & - & - & - & - & - & - & & - & - \\
\hline 5 & $9 \cdot 80$ & $0 \cdot 139^{*}$ & $10 \cdot 10$ & $0.090^{*}$ & $12 \cdot 20$ & $0.097^{*}$ & $13 \cdot 10$ & $0 \cdot 133^{*}$ & $0 \cdot 105^{*}$ & 13.85 & $0 \cdot 156^{*}$ & 14.79 & $0 \cdot 123^{*}$ & $0.080^{*}$ & 18.06 & $0.092 *$ & $0.059^{*}$ \\
\hline 10 & - & $\cdot 128$ & - & 0.077 & $10 \cdot 96$ & 0.070 & $12 \cdot 71$ & $0 \cdot 106$ & - & $13 \cdot 61$ & $0 \cdot 148$ & $14 \cdot 11$ & $0 \cdot 108$ & 0.092 & $17 \cdot 41$ & 0.081 & 0.086 \\
\hline 15 & - & $0 \cdot 129$ & $10 \cdot 04$ & 0.078 & $10 \cdot 32$ & 0.083 & - & $0 \cdot 116$ & - & $13 \cdot 43$ & $0 \cdot 143$ & $13 \cdot 71$ & $0 \cdot 137$ & - & $15 \cdot 13$ & $0 \cdot 117$ & - \\
\hline 20 & - & $0 \cdot 144$ & - & 0.079 & $10 \cdot 30$ & 0.099 & $12 \cdot 42$ & $0 \cdot 114$ & - & $13 \cdot 41$ & - & $13 \cdot 21$ & $0 \cdot 164$ & $0 \cdot 125$ & 12.76 & $0 \cdot 179$ & $0 \cdot 117$ \\
\hline 25 & $9 \cdot 70$ & 132 & - & 0.075 & - & $0 \cdot 101$ & - & $0 \cdot 141$ & - & $13 \cdot 25$ & - & $12 \cdot 35$ & $0 \cdot 107$ & $0 \cdot 129$ & $12 \cdot 62$ & 0.228 & $0 \cdot 182$ \\
\hline 30 & - & 0.092 & $9 \cdot 98$ & 0.088 & $10 \cdot 25$ & 0.099 & $12 \cdot 35$ & $0 \cdot 141$ & $0 \cdot 106$ & $11 \cdot 82$ & - & $11 \cdot 85$ & 0.081 & $0 \cdot 129$ & $12 \cdot 60$ & $0 \cdot 196$ & - \\
\hline 35 & - & $0 \cdot 103$ & - & $0 \cdot 119$ & - & $0 \cdot 104$ & $12 \cdot 26$ & $0 \cdot 166$ & - & - & - & - & - & $0 \cdot 126$ & - & - & $0 \cdot 196$ \\
\hline 40 & - & $0 \cdot 115$ & - & $0 \cdot 121$ & - & $0 \cdot 122$ & $11 \cdot 83$ & - & $0 \cdot 122$ & $11 \cdot 72$ & - & 11.82 & - & - & $12 \cdot 54$ & - & - \\
\hline 45 & - & $0 \cdot 108$ & - & $0 \cdot 114$ & - & $0 \cdot 100$ & - & - & - & - & - & - & - & $0 \cdot 110$ & - & - & $0 \cdot 163$ \\
\hline 50 & - & $0 \cdot 135$ & - & $0 \cdot 115$ & - & $0 \cdot 091$ & $11 \cdot 24$ & - & $0 \cdot 115$ & 11.72 & - & - & - & - & $12 \cdot 46 \dagger$ & - & $0 \cdot 142$ \\
\hline 55 & - & $0 \cdot 129$ & - & $0 \cdot 109$ & - & 0 . & - & - & - & - & - & - & - & $0 \cdot 117$ & - & - & $0 \cdot 155$ \\
\hline 60 & - & - & - & $0 \cdot 100$ & - & 0.085 & - & - & - & - & - & - & - & - & - & - & - \\
\hline 65 & - & - & - & 90 & - & 30 & - & - & - & - & - & - & - & - & - & - & - \\
\hline 70 & - & - & $9 \cdot 95$ & - & $10 \cdot 25$ & - & $11 \cdot 24$ & - & - & 11.72 & - & 11.78 & - & - & $12.92 \dagger$ & - & - \\
\hline
\end{tabular}




\begin{tabular}{|c|c|c|c|c|c|c|c|c|c|c|c|c|c|c|c|}
\hline Ser. & & $\begin{array}{c}37 \\
9 / 8 / 28\end{array}$ & 38 & & $\begin{array}{c}39 \\
29 / 8 / 28\end{array}$ & 40 & & 41 & & $\begin{array}{l}42 \\
/ 28\end{array}$ & $30 / 1$ & $\begin{array}{c}44 \\
1 / 28\end{array}$ & & $\begin{array}{c}45 \\
2 / 1 / 29\end{array}$ & 46 \\
\hline m. & $\mathrm{t}^{\circ} \mathrm{C}$ & $\lambda(\mathrm{L})$ & $\lambda(\mathrm{K})$ & $\mathrm{t}^{\circ} \mathrm{C}$. & $\lambda(\mathrm{L})$ & $\lambda(\mathrm{K})$ & $t^{\circ} \mathrm{C}$. & $\lambda(\mathrm{K})$ & $\mathrm{t}^{\circ} \mathrm{C}$ & $\lambda(L)$ & $t^{\circ} \mathrm{C}$ & $\lambda(J)$ & $\mathrm{t}^{\circ} \mathrm{C}$. & & $\lambda(J)$ \\
\hline 0 & $16 \cdot 7$ & - & - & $15 \cdot 9$ & - & - & $16 \cdot 8$ & - & $14 \cdot 95$ & - & $12 \cdot 2$ & - & $10 \cdot 5$ & - & - \\
\hline 2.5 & - & - & - & - & - & - & - & - & - & - & - & - & - & - & - \\
\hline 5 & $16 \cdot 54$ & $0 \cdot 196^{*}$ & $0 \cdot 170^{*}$ & $15 \cdot 68$ & $0 \cdot 126^{*}$ & $0 \cdot 100^{*}$ & $16 \cdot 62$ & $0.086^{*}$ & $14 \cdot 73$ & $0 \cdot 166^{*}$ & $12 \cdot 17$ & $0 \cdot 156^{*}$ & $10 \cdot 70$ & $0 \cdot 127^{*}$ & $0 \cdot 138^{*}$ \\
\hline 10 & $16 \cdot 45$ & $0 \cdot 168$ & $0 \cdot 194$ & $15 \cdot 68$ & $0 \cdot 119$ & - & $16 \cdot 46$ & 0.076 & $14 \cdot 73$ & $0 \cdot 129$ & - & $0 \cdot 125$ & - & $0 \cdot 101$ & - \\
\hline 15 & $16 \cdot 31$ & $0 \cdot 206$ & - & 15.09 & $0 \cdot 104$ & 0.090 & $16 \cdot 32$ & 0.084 & $14 \cdot 71$ & $0 \cdot 128$ & - & $0 \cdot 117$ & $10 \cdot 72$ & 0.097 & $0 \cdot 106$ \\
\hline 20 & 15.91 & - & $0 \cdot 178$ & $13 \cdot 61$ & $0 \cdot 164$ & $0 \cdot 134$ & $15 \cdot 55$ & 0.092 & - & $0 \cdot 131$ & - & $0 \cdot 103$ & - & - & - \\
\hline 25 & $13 \cdot 41$ & - & $0 \cdot 065$ & $13 \cdot 58$ & $0 \cdot 127$ & - & $13 \cdot 76$ & 0.092 & $14 \cdot 72$ & $0 \cdot 123$ & $12 \cdot 17$ & $0 \cdot 100$ & - & - & 0.082 \\
\hline 30 & $12 \cdot 96$ & - & $0 \cdot 054$ & $13 \cdot 41$ & 0.068 & $0 \cdot 138$ & $13 \cdot 68$ & $0 \cdot 100$ & - & $0 \cdot 126$ & - & $0 \cdot 117$ & - & - & - \\
\hline 35 & - & - & $0 \cdot 098$ & - & - & - & - & $0 \cdot 112$ & - & $0 \cdot 106$ & - & - & - & - & - \\
\hline 40 & 12.92 & - & $0 \cdot 112$ & 12.92 & - & $0 \cdot 103$ & $13 \cdot 55$ & $0 \cdot 117$ & - & - & - & $0 \cdot 141$ & $10 \cdot 72$ & - & - \\
\hline 45 & - & - & $0 \cdot 129$ & - & - & - & - & - & - & - & - & $0 \cdot 123$ & - & - & - \\
\hline 50 & 12.92 & - & - & $12 \cdot 78$ & - & $0 \cdot 108$ & - & - & $14 \cdot 72$ & - & $12 \cdot 17$ & $0 \cdot 082$ & - & - & - \\
\hline 55 & - & - & - & - & - & - & - & 0.096 & - & - & - & - & - & - & - \\
\hline 60 & - & - & - & - & - & - & $13 \cdot 51$ & - & - & - & - & - & - & - & - \\
\hline 65 & - & - & - & - & - & - & - & - & - & - & - & - & - & - & - \\
\hline 70 & $12 \cdot 92$ & - & - & $12 \cdot 76$ & - & - & $13 \cdot 53$ & - & $14 \cdot 71$ & - & $12 \cdot 17$ & - & $10 \cdot 72$ & - & - \\
\hline
\end{tabular}


Previous work on the phosphate content of sea-water has shown that at some time during each spring there is a very rapid diminution, indicating that the multiplication of the diatoms has used up the phosphate. Accordingly, one might expect that the upper layers of the sea would offer greater obstruction to the passage of light immediately after this outburst of diatoms than they did before it. Table VII shows that in 1928 the outburst occurred between April 19th and May 2nd, but the values of $\lambda$ in Table VI prove that the very clear water present on April 19th persisted through early May. Again, on July 11th and 23rd, the first 15 metres was clear water, whereas on August 9th the clear water was deeper. The phosphate content was, however, closely the same on these three dates.

\section{TABLE VII.}

Phosphate in Sea-water, as mg. $\mathrm{P}_{2} \mathrm{O}_{5}$ Per $\mathrm{m}^{3}$, at Station E1, 1928.

$\begin{array}{rcccccccc}\text { m. } & \text { Mar. 27 } & \text { April 5 } & \text { April 19 } & \text { May 2 } & \text { May 7 } & \text { July 11 } & \text { July 23 } & \text { Aug. 9 } \\ 0 & 28 & 24 & 24 & 10 & 9 & 8 \cdot 5 & 0 & 0 \cdot 5 \\ 5 & 25 & 25 & 23 & 10 & 9 & 1 \cdot 5 & 0 & 0 \\ 10 & - & - & - & - & 10 & 2 & 1 \cdot 5 & 0 \\ 15 & 25 & - & - & 10 & 22 & 2 & 1 \cdot 5 & 0 \cdot 5 \\ 20 & - & - & - & 17 & 18 & 4 & 10 \cdot 5 & 1 \\ 25 & 26 & - & - & 18 & 18 & 8 \cdot 5 & 15 & 10 \\ 30 & - & - & - & - & 19 & 20 & 13 & 13 \\ 40 & - & - & - & - & - & 19 & 13 & -12 \\ 50 & - & 26 & - & - & - & - & 14 & 12 \\ 70 & - & 27 & 23 & 20 & 19 & 24 & 14 & 19\end{array}$

In the course of the study of the seasonal changes in phosphate at Station E1, extending from 1923 onwards, the conclusion has been reached that the water in this region is but little subject to influx of water from other sources, or, to be more precise, that the water which reached this position had been subjected to similar changes in its phosphate condition. The possibility, however, that some of the changes in the values of $\lambda$ may be occasioned by the influx of water of different origin must be considered. Table VIII records the salinity at Stations E1, E2, mid-Channel, and E3, off Ushant, from June, 1927, to January, 1929. As a rule the water is of greater salinity at E3 than it is at E2, and the salt content of E2 is usually greater than that of E1. The analyses given were carried out at the Government Chemist's Laboratory, London. It may be concluded that from June up to November, 1927, there was no appreciable movement of water. On December 9th, however, the rather higher salinity indicates movement of water from near E2 towards E1, and this coincides with a clearing of the water. In February, 1928, an abnormal condition obtained, inasmuch as the salinity of the water decreased from E1 to E3. Such a condition was only observed once 


\section{TABLE VIII.}

Salinity at Stations E1, 10 miles S.W. of the Eddystone, E2, MidChannel, and E3, off Ushant, in parts Per 1000; Averaged for the Water Column to Indicate Movement of Water TOWARDS E1.

1927

$1927 \quad 1928$

1928

June 27 Aug. 2 Sept. 7 Oct. 3 Nov. 4 Dec. 9 Jan. 31 Feb. 21 Mar. 27 Apl. 19 May 2

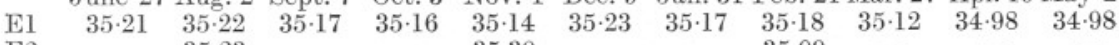

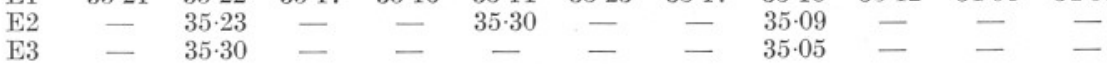

1928

1929

May 8 June 4 July 4 July 23 Aug. 16 Aug. 29 Sept. 18 Oct. 2 Nov. 30 Jan. 2 Jan. 7

$\begin{array}{lccccccccccc}\text { E1 } & 34.95 & 34.90 & 34.93 & 34.92 & 34.90 & 34.91 & 35.07 & 35.02 & 35.32 & 35.35 & 35.39 \\ \text { E2 } & 34.90 & - & - & - & 35.29 & - & - & - & - & - & 35.36\end{array}$

E2 $34.90 \quad-\quad-\quad-$\begin{tabular}{llllll}
$35 \cdot 29$ & - & - & - & - & - \\
\hline
\end{tabular}

\section{TABLE IX.}

Rainfall for Five Stations in Basin of R. Seine and for Six in Basin of R. LoIRe, gIVEN as mm. PER MONTH.

SEINE

Chaumont

Chaumont-en-Verin

Paris (Parc St. Maur)

Chartres

Rouen

Total

Gross total

LoIre

Tours

Orleans (École)

Chateauroux

Poitiers

Le Mans (École)

Nantes

Total

Gross total

Fercentages of normal.

Seine Total

Gross total

Loire

$$
\overbrace{\text { Jan. Feb. Mar. Jan. Feb. Mar. }}^{\text {Normal. }}
$$

$\begin{array}{ll}66 & 55 \\ 50 & 38 \\ 39 & 31\end{array}$

$50 \quad 38$

39

40

53

250

32

65

65

44

44

42

42
48

$197 \quad 240$

45

53

40

40
34

34
71

$24:$

- $\quad-687$

$\begin{array}{lll}56 & 48 & 59\end{array}$

$43 \quad 33$

$51 \quad 41$

$51 \quad 39$

$54 \quad 39$

$71 \quad 52$

$326 \quad 252$

298
$-\quad 876$

$\begin{array}{rrrrrrrrr}- & - & - & 98 & 193 & 129 & 150 & 187 & 121 \\ - & - & - & - & - & 136 & - & - & 150 \\ - & - & - & 96 & 207 & 187 & 142 & 162 & 163 \\ - & - & - & - & - & 159 & - & - & 155\end{array}$

1928

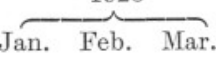


before since 1921, namely, in 1923, and the rainfall for that area of France drained by the rivers Seine and Loire was abnormally high during the first three months of that year, and for 1928, as shown in Table IX. Accordingly, we may suppose that the water of the rivers opening on to the Atlantic coast of France was responsible for the abnormal salinity conditions found. The high salinity water persisted around E1, up to March 27th, but on April 19th and early in May less saline water was found at E1. To this influx of mid-Channel water, deduced from the data of Table VIII and the known water movements of the Channel, we must attribute the exceptionally great clearness found from April 19th onwards.

It might be thought that the change in the phosphate content, attributed previously to the diatom outburst, was in reality due to this influx of water from near mid-Channel. There are good reasons, however, for rejecting this view. Firstly, the fact that the change in salinity had already taken place by April 19th, whereas the drop in phosphate content did not take place until after that, and, secondly, that, as shown in Table $\mathrm{X}$, the phosphate content from February to May was very similar at all three stations.

\section{TABLE $\mathrm{X}$.}

Phosphate in Sea-water, as mg. $\mathrm{P}_{2} \mathrm{O}_{5}$ Per m. ${ }^{3}$, at Stations E1, E2, AND E3, YEAR 1928.

Large asterisk indicates that bottom has been reached before the depth indicated.

$\begin{array}{rcccccc}\text { Date } & \text { February 21 } & 21 & 22 & \text { May 7 } & 8 & 9 \\ \text { m. } & \text { E1 } & \text { E2 } & \text { E3 } & \text { E1 } & \text { E2 } & \text { E3 } \\ 0 & 27 & 11 & 23 & 9 & 7 & 10 \\ 5 & 27 & 24 & - & 9 & 7 & - \\ 10 & - & - & - & 10 & 8 & 13 \\ 15 & - & - & - & 22 & 17 & 18 \\ 20 & - & - & - & 18 & 20 & 25 \\ 25 & -- & - & - & 18 & - & 26 \\ 30 & - & - & - & 19 & 19 & - \\ 70 & 28 & - & - & 19 & - & - \\ 80 & * & 25 & - & * & 21 & - \\ 105 & & * & 25 & & * & 28\end{array}$

This definite connection between the sudden clearing of the water in the spring of 1928 and the variation in salinity will not, however, explain all the variations of $\lambda$. It does, however, appear to be the cause of the unusual clearness of the water found at E1 on September 18th, 
as shown by the sudden rise in salinity, denoting inflow from near mid-Channel. We are, therefore, faced with the necessity of accounting for the existence of layers of relatively great opacity at different levels, even on the same day and within short periods of time, during which there are no indications of any water movements. For these we may tentatively suggest the vertical movements of the zooplankton as a cause. It is true that zooplankton are fewer in numbers and individually larger than the phytoplankton; they are, however, far more capable of aggregation. Russell $(\mathbf{5}, \mathbf{6})$ has shown in a series of papers that the various members of the zooplankton are distributed at depths depending upon the intensity of the illumination at the time, and upon the duration of that illumination. At dusk the animals tend to be found near the surface. During the night they become more uniformly distributed, and at dawn they are again attracted towards the surface. With the increasing light intensity they move downwards, so that the depth at which they are found depends, not only upon the species and the degree to which they are positively or negatively phototropic, but also upon the time at their disposal for travelling under the influence of the stimulus. It becomes of interest, therefore, to see whether any relation exists between the actual intensity of the light, and the depth at which the maximum value of $\lambda$ occurs in each series. Table XI contains the relevant data. Values for $\beta$ have also been included as indicating whether the day as a whole was dull or of moderate or intense sunshine. It may be seen that the values of the vertical illumination $\mathrm{V}$ vary fairly widely, but are, as a rule, to be reckoned in thousands of metre candles, and it is remarkable that, whereas in the winter, autumn, and early spring the maximum value of $\lambda$ is usually found at about 5 metres, yet in late spring and summer it may be found at from 15 to 40 metres. In this connection the results obtained in July and August, 1928, are especially significant. The maximum value of $\lambda$ was found at 10 and 15 metres on August 9th, a dull day, whereas on July 11th it was found between 20 and 30 metres, on July 23rd, between 25 and 35 metres, and on August 29th, from 20 to 30 metres. These were bright sunny days. There appears, therefore, to be some evidence for considering that the layers in which light absorption is a maximum are those in which the photic stimulus has resulted in an aggregation of the zooplankton. This possibly can, of course, be tested by direct experiment, and it is hoped to do this.

In conclusion, we desire to express our thanks to Captain V. Lord and the crew of the Salpa for their great assistance in the transport and handling of the instruments, often under conditions of considerable difficulty, the lowering of the photometers in rough water being no easy matter. Though in use since 1924, none of the apparatus has ever been 
TABLE XI.

The values of $\lambda$ shown are maxima or specially high values for the various series and occurred at the depth, in metres, shown. $\mathrm{V}$ denotes the vertical illumination, in thousands of metre candles, at depth tabulated.

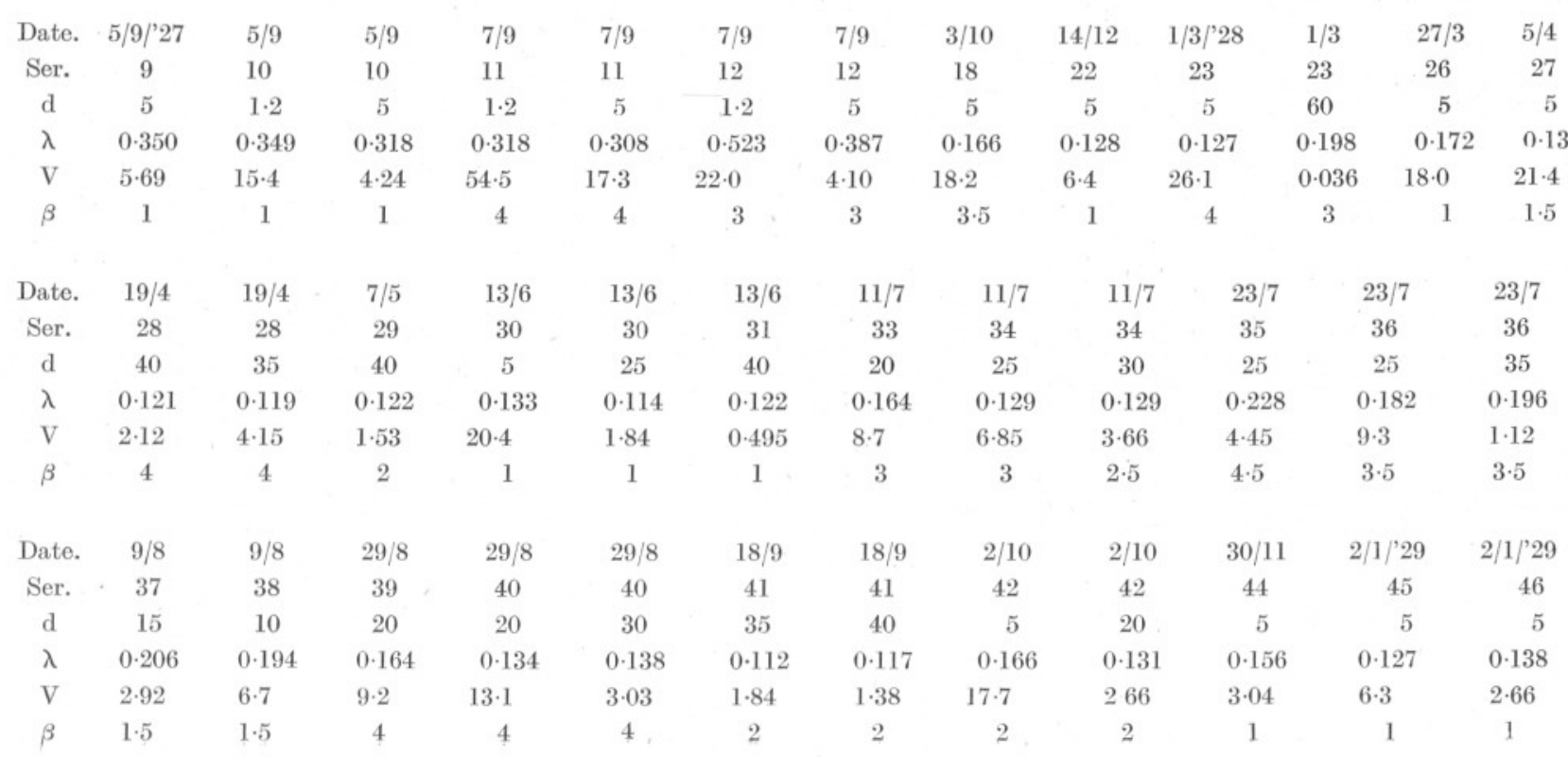


broken or damaged at sea. We desire also to record our indebtedness to the Royal Dublin Society for facilities connected with the construction and standardising of the photometers and to the Marine Biological Association for the major part of the apparatus and for laboratory and sea-going facilities. Our thanks are also due to the Director and Staff of the Research Laboratories of the General Electric Company for their valuable advice, and to Mr. S. G. Monk for assisting in testing for troubles associated with damp weather.

\section{SUMMARY.}

Measurements were made, throughout the year 1928, of the penetration of daylight into sea-water in the English Channel. One vacuum and two gas-filled potassium photo-electric cells were used below water. In all save the first series an efficient diffusing surface was used with the photometers so that the corrections for obliquity of illumination, necessary in our earlier work, were rendered almost negligible. From October onwards a more sensitive gas-filled cell was available for use, together with a more powerful amplifier and a more efficient insulation of the high-tension batteries; improvements in the switching of the photometers on and off were also incorporated.

The following conclusions have been reached :-

1. The conclusions put forward tentatively in 1928, concerning the agreement between the vacuum and gas-filled cells, have been substantiated in the main, although the vacuum photometer, which is relatively more sensitive to the blue end of the spectrum, generally indicated rather higher opacities.

2. There are no regular seasonal changes in the opacity of the water. A very high opacity occurred in the autumn of 1927, but this was not repeated in 1928 .

3. On one occasion, in April 1928, an unusual degree of clearness of the water has been shown to have been associated with the influx of water of lesser salinity, from further out in the Channel. The clearness of the water at E1 in December, 1927, and September, 1928, is probably due to an influx of clearer water, though the salinity changes indicating this are not so well marked.

4. The main spring diatom outburst in 1928 took place when the water at E1 was exceptionally clear and failed to produce any very noticeable obscuring effect.

5 . Changes in the level at which the maximum values of $\lambda$ occur may be observed in the course of a day and are well marked within the 
fortnightly periods of observation. Further evidence has been accumulated in favour of the view, put forward tentatively in 1928, that these changes are due to alterations in the level at which the zooplankton has become aggregated during the execution of phototropic movements. Direct evidence is lacking on this point, but it is hoped that it may be obtained this year.

6. For Station E1 the mean values of the percentages of the light in air reaching 20,40 , and 60 metres were $6 \cdot 62,0 \cdot 72$, and 0.085 respectively ; for depths of $0-20,20-40,40-60$ metres the mean values of $\lambda$ were $0 \cdot 150$, $0 \cdot 120$, and $0 \cdot 111$. The most turbid water was found at $25 \mathrm{~m}$. on July $23 \mathrm{rd}$, $\lambda$ being $0 \cdot 228$, with very clear water above, $\lambda 0.081$ at $10 \mathrm{~m}$. The clearest water column was on April 19th, $\lambda 0 \cdot 077-0 \cdot 121$, though on August 9th at 25 and $30 \mathrm{~m}$. values of $\lambda 0.065$ and 0.054 respectively were obtained.

7. Measurements down to $25 \mathrm{~m}$. showed that the horizontal light (viz. that falling on a vertical surface) was from 0.61 to 0.47 , mean 0.54 of the vertical light. The value is higher than that formerly obtained without an efficient diffusing surface on the photometer.

8. The illumination at the depth (D) at which the Secchi disc is just visible is around $16 \%$ of that in air. Very roughly the opacity may be found thus, $\lambda_{0-20}=1 \cdot 7 / \mathrm{D}$. The visibility of the disc is independent of the illumination in air, within wide limits.

\section{REFERENCES.}

1. Poole, H. H. On the Photo-electric Measurement of Submarine Illumination. Sci. Proc. Royal Dublin Soc., 1925 18, 99-115.

2. Poole, H. H., and Atrins, W. R. G. On the Penetration of Light into Sea Water. J. Marine Biol. Assoc., 1926, 14, 177-198.

3. Poole, H. H., and Atkins, W. R. G. Further Photo-electric Measurements of the Penetration of Light into Sea Water. J. Marine Biol. Assoc., 1928, 15, 455-483.

4. Poole, J. H. J. A simple form of photo-electric photometer using a neon lamp to measure the current. Sci. Proc. Royal Dublin Soc., $1928,19,17-25$.

5. Russelu, F. S. The Vertical Distribution of Marine Macroplankton. Parts I-VI. J. Marine Biol. Assoc., 1925, 13, 769-809 ; 1926, 14, $101-159$; 1926,14, 387-414; 1926, 14, 415-440 ; 1927, 14, 557-608; $1928,15,81-99$.

6. Russeld, F. S. The Vertical Distribution of Plankton in the Sea. Biological Reviews, 1927, 2, 231-262. 